\title{
Dynamic Computation of Flexible Multibody System with Uncertain Material Properties
}

by

Jinglai Wu ${ }^{1,2}$, Zhen Luo $^{2}$, Nong Zhang ${ }^{2}$, Yunqing Zhang ${ }^{*}$

${ }^{1}$ National Engineering Research Centre for CAD

Huazhong University of Science \& Technology, Wuhan, Hubei 430074, China

2 School of Electrical, Mechanical and Mechatronic Systems

The University of Technology, Sydney, NSW 2007, Australia

Original submission: 11 February, 2016

${ }^{*}$ Correspondence author in the process of manuscript submission

(Prof. Y. Zhang, Email: zhangyq@hust.edu.cn)

This paper is submitted for possible publication in Nonlinear Dynamics. It has not been previously published, is not currently submitted for review to any other journals, and will not be submitted elsewhere during the peer review. 


\begin{abstract}
Based on the theory of Absolute Nodal Coordinate Formulation (ANCF), this paper proposes a new dynamic computation method to solve the flexible multibody system with uncertain material properties (Young's modulus and Poisson's ratio) that may be induced by the material asymmetric distribution. Rather than traditionally considering an uncertain factor as one single variable in the whole system, the material properties vary continuously in the space domain so that they are described by the random field, which is then discretized to countable random variables using the expansion optimization linear estimation (EOLE) method. The uncertain response of the system is approximated by the Polynomial Chaos (PC) expansion, numerically implemented by a collocation method. We propose and prove an important theory that the collocation method provides the same results as the Gaussian quadrature formula if the roots of the corresponding orthogonal polynomials are used as the collocation points. As a result, the proposed method is a non-intrusive technique that does not modify the original solver but only adds a pre-process and a post-process. The uncertain displacement of system is finally illustrated by an ellipse and ellipsoid, which visually show the uncertainty extent and correlation between different coordinates. The numerical examples show that the proposed method has almost an equivalent accuracy of Monte Carlo simulation but much higher efficiency.
\end{abstract}

Keywords: flexible multibody system; ANCF; random field; PC expansion; collocation method

\title{
1. Introduction
}

The multibody systems are quite important for the machine, automotive, robot, aerospace system, engineering vehicle, and many other industries depending on the mechanical engineering. The rigid multibody systems have been well studied [1], so there have been more and more studies to solve the flexible multibody systems in recent decades, which are more satisfied with some practical application conditions. Traditional finite element methods used to solve the flexible multibody system are based on the assumption that only small deformation and small rotation are happened in the components. Therefore, these methods will not provide proper dynamic results when the components of a system are subject to large rotation and large deformation [2]. 
To solve the problem of large deformation and rotation, Shabana [3] proposed a new Absolute Nodal Coordinate Formulation (ANCF) to model the element of flexible components. The ANCF defines the element coordinates as the absolute displacements and global slopes rather than the infinitesimal and finite rotations in traditional finite element methods [4]. As a result, the locations and deformations of the material points in the model are defined in the global coordinate system, using the element shape function and the nodal coordinates, which leads to that the mass matrix of the system equations remains constant and the centrifugal and Coriolis forces are identically equal to zero [5]. As a non-incremental finite element method, the ANCF has been considered as a benchmark approach in the research of flexible multibody dynamics [6], especially for the large deformation flexible multibody systems [7-9].

There have been some applications for solving the flexible multibody systems by using the ANCF. Zhang et al. [10] investigated the viscoelastic flexible multibody systems with a fractional constitutive model by using the ANCF. Tian et al. [11] proposed a novel methodology for modeling and analysis of flexible multibody system with clearance and lubricated revolute joints by using the ANCF. The ANCF was also incorporated with the Natural Coordinate Formulation (NCF) to build the dynamic model of the rigid-flexible multibody systems by Tian et al. [12], who derived a new general and comprehensive approach for a spatial flexible multibody system with cylindrical clearance joint. Liu et al. [13] used the ANCF-NCF combined method to compute the dynamic response of a large scale rigid-flexible multibody system composed of composite laminated plates. More applications about the ANCF in multibody systems can be found in references [14-17].

The studies mentioned above are based on the deterministic assumption, which means that all the parameters in the governing equations are thought to be exactly known. However, a multibody system contains many uncertain factors, e.g. the geometry size of a component has a tolerance to manufacturing easily, which will contribute to the uncertainty of mass, gravity force, and constraints; the different kinds of raw material and manufacturing process may lead to the inhomogeneous distribution of material, which will further lead to the change of material properties including the Young's modulus, Poisson's ratio, and density. Since the uncertainty of material properties is induced by the inhomogeneous distribution of material in space, they may continuously change in the space domain, which are widely existed in the flexible components. These uncertain factors may lead to a quite different response compared to the deterministic response. To compute the dynamical response of a multibody system more accurately, it is demanded to take account the uncertain factors. 
The uncertainty can be divided into two types that are the aleatory uncertainty and epistemic uncertainty $[18,19]$. Aleatory uncertainty, also termed as objective or stochastic uncertainty, describes the inherent variation associated with a physical system or environment. Epistemic uncertainty or subjective uncertainty, on the other hand, derives from some level of ignorance or incomplete information about a physical system or environment. The aleatory uncertainty is commonly expressed by the random variables (or random field) while the epistemic uncertainty may be described as the interval variables or convex models [20] and so on.

When the information of uncertain factors is limited, the interval variables are usually used to describe the uncertainty, because they only need the bounds information of uncertain variables. Thus, typically the uncertainty of the geometry size is quite suitable to be described by the interval variables. The bounds of the response can be obtained by using optimization methods [21] or interval arithmetic [22]. To save the computational cost, the interval arithmetic is usually used to produce an envelope of the actual response. There have been a few studies using the interval method to solve the multibody system with uncertainty. $\mathrm{Wu}$ et al. [23] employed the Chebyshev series expansion method to solve the rigid multibody system, where the geometry size and the external torque are considered as interval parameters. Wang et al. [24] also used the same method to solve the rigid-flexible multibody system with interval uncertain parameters, which was the first time that interval parameters are introduced into the rigid-flexible multibody system. The OpenMP directives were used to parallelize the computation of the nonlinear algebraic equations with deterministic sampling parameters, which improved the computational efficiency largely.

The material properties of a component in the flexible multibody system vary continuously in the space domain, which means that the uncertainty has to be characterized by using the concept of 'field'. We consider that the material properties change in the space domain rather than in the time domain, i.e. the Young's modulus or Poisson's ratio at different location of a component is different, but they will not change in the simulation process. As the authors' known, there has no definition about the 'interval field' so that we cannot use interval to describe the continuous uncertainty of material properties. In the stochastic finite element method, the random field that handles randomness and spatial variability simultaneously has been used to describe the uncertainty of material properties. Therefore, the random field can be employed to characterize the uncertain material properties in the dynamic computation of the flexible multibody system. The continuous random field is formally defined as an indexed set of random variables, the index belonging to some continuous uncountable set. The random field can be 
approximated as closely as desired by restricting the index to a set dense in the indexing set. Stretching mathematical rigor further, a random field can be represented by its values at a discrete set of points in its domain of definition [25], which means that a continuous random field can be approximated represented by a set of countable random variables. Since the uncountable index set is not convenient to implement in the numerical computation, the random field has to be discretized to countable random variables.

The discretization methods for the random field can be divided into three groups that are point discretization, average discretization, and series expansion methods [26]. The point and average discretization methods involved a finite number of random variables having a straightforward interpretation: point values or local averages of the original field. These random variables can be expressed as weighted integrals of random field over the volume of the system. Using these random variables, the random field can be approximated by a finite summation of the product of these random variables and some deterministic functions or basis on the space domain. However, the basis used in these methods is not optimal, because these bases have a compact support. The series expansion methods aim at expanding any realization of the original random field over a complete set of deterministic functions [26]. The discretization occurs thereafter by truncating the obtained series after a finite number of terms. The series expansion methods mainly include the Karhunen-Loeve (K-L) expansion [25], orthogonal series expansion (OSE) [27], and expansion optimal linear estimation (EOLE) method [28]. Reference [26] provided a detailed comparison of these discretization methods, and some conclusions are given as follows: the K-L expansion is the best choice for the exponential autocorrelation function and the accuracy of EOLE is very close to it, but the K-L expansion may not be fit to handle the exponential square autocorrelation function; the EOLE provides better accuracy than the OSE for both types of autocorrelation functions.

After the random field is discretized to countable random variables, many probabilistic methods can be employed to solve the propagation of probabilistic uncertainty. The probabilistic methods can be broadly classified into two major categories: statistical methods and non-statistical methods. Monte Carlo method [29] is one of the most widely used statistical methods, which is easy to implement. The Monte Carlo method takes samples of the random variables (input) based on their probability distribution and then computes the probability distribution of response (output) directly. The accuracy of Monte Carlo method depends on the sampling size, in accordance with the weak law of large number. Therefore, to get enough high accuracy, it usually requires a large number of sampling points. For the dynamical computation of a 
flexible multibody system, each running takes a large amount of time especially for the large-scale system, so that the thousands of running for Monte Carlo method cannot be affordable. As a result, the Monte Carlo method is often used as the reference of other methods.

To reduce the computational cost, the non-statistical methods can be used, e.g. the perturbation method, Neumann expansion method, and Polynomial Chaos (PC) expansion method [30]. Both the perturbation method and Neumann expansion method requires the small uncertainty extent of random variables while the PC expansion method is quite fit for the problems with large uncertainty extent variables. The PC expansion method approximates the response of system by a truncated orthogonal series and then uses the characteristics of orthogonal polynomials to estimate the first and second moment of the random response. The PC expansion methods have been widely used in various engineering problems, such as in fluid mechanics [31], vehicle dynamics [32, 33], structure dynamics [34], and optimization problems [35].

There have been only a few studies investigating the random uncertainty of the multibody systems. Soize et al. [36] and Schmitt et al. [37] used the Monte Carlo method to handle the rigid multibody systems under random uncertainty. Sandu et al. [38, 39] used the PC expansion method to investigate the uncertainty of rigid multibody systems. Wang et al. [40] used both the perturbation method and Monte Carlo method to investigate the dynamic response of flexible multibody systems with parameter uncertainty, but it was based on the assumption of small deformation. In summary, there has no publication studying the large deformational flexible multibody system with random uncertainty, especially for using the random field to characterize the uncertainty of material properties.

This paper studies the dynamic response of the flexible multibody system with uncertain material properties, in which the Young's modulus and Poisson's ratio are considered as random field. The paper is organized as follows. Section 2 gives a brief review about the theory of ANCF, and then the random field and its discretization are provided in Section 3. Section 4 provides the PC expansion theory and its numerical implementation method. The detailed computational scheme will be given in Section 5, and two numerical examples are provided in Section 6 to demonstrate the validation of the proposed method. The conclusion is given in the last section. 


\section{ANCF-based beam element}

\subsection{Planar and spatial ANCF-based beam element}

The two-dimensional [41] and three-dimensional [42] ANCF-based beam element are shown as Fig. 1 and Fig.2, respectively. The displacement field of two-dimensional shear deformable beam element can be defined in the global coordinate system as

$$
\mathbf{r}=\left[\begin{array}{ll}
r_{1} & r_{2}
\end{array}\right]^{\mathrm{T}}=\mathbf{S e}, \mathbf{e}=\left[\begin{array}{ll}
\mathbf{e}_{i}^{\mathrm{T}} & \mathbf{e}_{j}^{\mathrm{T}}
\end{array}\right]^{\mathrm{T}}=\left[\begin{array}{llllll}
\mathbf{r}_{i}^{\mathrm{T}} & \mathbf{r}_{i, x}^{\mathrm{T}} & \mathbf{r}_{i, y}^{\mathrm{T}} & \mathbf{r}_{j}^{\mathrm{T}} & \mathbf{r}_{j, x}^{\mathrm{T}} & \mathbf{r}_{j, y}^{\mathrm{T}}
\end{array}\right]^{\mathrm{T}}
$$

while the displacement of the three-dimensional beam element is given as

$$
\mathbf{r}=\left[\begin{array}{lll}
r_{1} & r_{2} & r_{3}
\end{array}\right]=\mathbf{S e}, \mathbf{e}=\left[\begin{array}{ll}
\mathbf{e}_{i}^{\mathrm{T}} & \mathbf{e}_{j}^{\mathrm{T}}
\end{array}\right]^{\mathrm{T}}=\left[\begin{array}{llllllll}
\mathbf{r}_{i}^{\mathrm{T}} & \mathbf{r}_{i, x}^{\mathrm{T}} & \mathbf{r}_{i, y}^{\mathrm{T}} & \mathbf{r}_{i, z}^{\mathrm{T}} & \mathbf{r}_{j}^{\mathrm{T}} & \mathbf{r}_{j, x}^{\mathrm{T}} & \mathbf{r}_{j, y}^{\mathrm{T}} & \mathbf{r}_{j, z}^{\mathrm{T}}
\end{array}\right]^{\mathrm{T}}
$$

where $\mathbf{r}$ denotes the nodal coordinates in the global coordinate system, $\mathbf{S}$ is the shape function, $\mathbf{e}$ denotes the absolute nodal coordinate vector of node $i$ and $j, \mathbf{r}_{p}(p=i, j)$ is the position coordinates defined in the global coordinate system, the $\mathbf{r}_{p, x}$ denotes the derivative of $\mathbf{r}_{p}$ with respect to local coordinate $x$, while the $\mathbf{r}_{p, y}$ and $\mathbf{r}_{p, z}$ have the similar meaning.



Figure. 1 Two-dimensional beam element

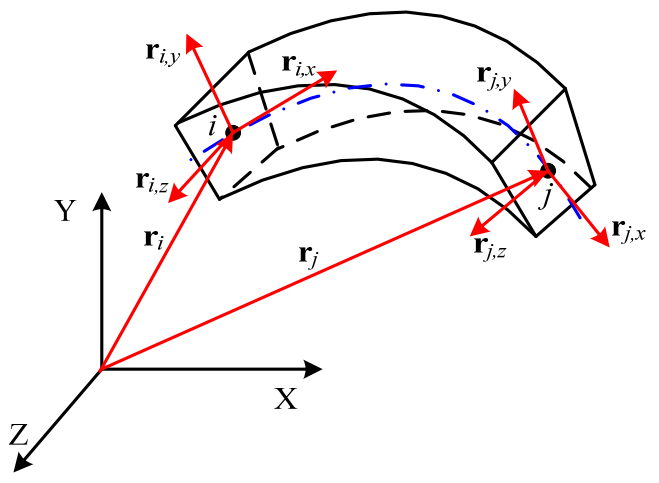

Figure. 2 Three-dimensional beam element

\subsection{Equations of motion}

By using Newton - Euler formulation, the element equations of motion can be written as

$$
\mathbf{M}_{e} \ddot{\mathbf{e}}=\mathbf{Q}_{e}-\mathbf{F}_{e}
$$

where the constant element mass matrix $\mathbf{M}_{e}$ can be computed by the following formula 


$$
\mathbf{M}_{e}=\int_{V} \rho_{e} \mathbf{S}^{\mathrm{T}} \mathbf{S} d V
$$

Here $\rho_{e}$ is the density of material, and $V$ denotes the volume of element. $\mathbf{F}_{e}$ denotes the element elastic force vector and $\mathbf{Q}_{e}$ is the element external generalized force vector that can be deduced by continuum mechanism approach $[43,44]$. To improve the computational efficiency, the invariant matrix method [43] can be used to compute the element elastic force, which is computed by the following equation.

$$
\mathbf{F}_{e}=-\mathbf{K}(\mathbf{e}) \mathbf{e}=-\left(\mathbf{K}_{1}+\mathbf{K}_{2}(\mathbf{e})\right) \mathbf{e}
$$

where the nonlinear stiffness matrix $\mathbf{K}(\mathbf{e})$ can be divided into summation of a constant stiffness matrix $\mathbf{K}_{1}$ and another nonlinear stiffness matrix $\mathbf{K}_{2}(\mathbf{e})$ which is depend on the generalized coordinates. The constant stiffness matrix of the two-dimensional element and three-dimensional element is expressed by

$$
\mathbf{K}_{1}^{2 D}=-(\kappa+G) \int_{V} \sum_{\alpha=1}^{2} \mathbf{S}_{, \alpha}^{\mathrm{T}} \mathbf{S}_{, \alpha} d V, \mathbf{K}_{1}^{3 D}=-\frac{3 \kappa+2 G}{2} \int_{V} \sum_{\alpha=1}^{3} \mathbf{S}_{, \alpha}^{\mathrm{T}} \mathbf{S}_{, \alpha} d V
$$

where the $G$ denotes the Young's modulus, and $\kappa$ is the Poisson's ratio. The entry of nonlinear stiffness matrix $\mathbf{K}_{2}(\mathbf{e})$ can be computed by

$$
\left[\mathbf{K}_{2}(\mathbf{e})\right]_{i j}=\mathbf{e}^{\mathrm{T}} \mathbf{C}_{\mathbf{K}_{2}}^{i j} \mathbf{e}
$$

where the $\mathbf{C}_{\mathbf{K}_{2}}^{i j}$ is a constant matrix, termed as invariant matrix, which is expressed as

$$
\begin{aligned}
\mathbf{C}_{\mathbf{K}_{2}}^{i j}= & \frac{\kappa+2 G}{2} \sum_{\alpha=1}^{N} \int_{V}\left(\mathbf{S}_{, \alpha}^{\mathrm{T}} \mathbf{S}_{, \alpha}\right)_{i}^{T}\left(\mathbf{S}_{, \alpha}^{\mathrm{T}} \mathbf{S}_{, \alpha}\right)_{j} d V \\
& +\frac{\kappa}{2} \sum_{\alpha=1}^{N} \sum_{\beta=1, \beta \neq \alpha}^{N} \int_{V}\left(\mathbf{S}_{, \alpha}^{\mathrm{T}} \mathbf{S}_{, \alpha}\right)_{i}^{T}\left(\mathbf{S}_{, \beta}^{\mathrm{T}} \mathbf{S}_{, \beta}\right)_{j} d V+G \sum_{\alpha=1}^{N} \sum_{\beta=1, \beta \neq \alpha}^{N} \int_{V}\left(\mathbf{S}_{, \alpha}^{\mathrm{T}} \mathbf{S}_{, \beta}\right)_{i}^{T}\left(\mathbf{S}_{, \alpha}^{\mathrm{T}} \mathbf{S}_{, \beta}\right)_{j} d V
\end{aligned}
$$

where $N=2$ for two-dimensional element and $N=3$ for three-dimensional element. It should be noted that the material properties, e.g. Young's modulus and Poisson's ratio, are considered as constant in the Eqs. (6) and (8).

\section{Material property represented by random field}

As aforementioned in the introduction, the material properties of flexible components are uncertain and they vary in the space domain continuously. Therefore, the random field is the best tool to describe the uncertain material properties. This Section will introduce the random field and its discretization. 


\subsection{Random field}

In elementary of probability theory outcomes of experiments are usually described in terms of "random variables", uncertain quantities to be observed. Similarly, it is meaningful to associate with the term "random field" a giant laboratory where an investigator can do numerous experiments. The location of each experimental setup in the laboratory is identified, in the terminology of random fields, by a set of coordinates or parameters [45]. For the $d$-dimensional space, locations are denoted by a vector $\mathbf{x} \in \mathbf{D}$, where $\mathbf{D}$ is an open set of $\mathrm{R}^{d}$ describing the system geometry. When $d=1$, the random field is called as univariate random field which is also termed as random process. Therefore, a random field $Y(\mathbf{x}, \theta)$ can be defined as a curve in $\mathcal{L}^{2}(\Omega, \mathcal{F}, \mathcal{P})$, that is a collection of random variables indexed by the continuous parameter $\mathbf{x}$. This means that for a given $\mathbf{x}_{0}, Y\left(\mathbf{x}_{0}, \theta\right)$ is a random variable. Conversely, for a given outcome $\theta_{0}, Y\left(\mathbf{x}_{0}, \theta_{0}\right)$ is a realization of the field [26].

The covariance of values of a random field at two different locations, $Y\left(\mathbf{x}_{1}\right)$ and $Y\left(\mathbf{x}_{2}\right)$ (short for $\left.Y\left(\mathbf{x}_{i}, \theta\right)\right)$, defines the covariance function ( of $\mathbf{x}_{1}$ and $\mathbf{x}_{2}$ ):

$$
C\left(\mathbf{x}_{1}, \mathbf{x}_{2}\right) \equiv \operatorname{Cov}\left[Y\left(\mathbf{x}_{1}\right), Y\left(\mathbf{x}_{2}\right)\right]=\mathrm{E}\left[Y\left(\mathbf{x}_{1}\right) Y\left(\mathbf{x}_{2}\right)\right]-\mathrm{E}\left[Y\left(\mathbf{x}_{1}\right)\right] \mathrm{E}\left[Y\left(\mathbf{x}_{2}\right)\right]
$$

where $\operatorname{Cov}[f, g]$ denotes the covariance of random variables $f$ and $g, E[f]$ represents the expectation or mean of random variable $f$. Using $\sigma(f)$ delegate the standard deviation of random variable $f$, the correlation function of random field is given as

$$
\rho\left(\mathbf{x}_{1}, \mathbf{x}_{2}\right) \equiv \frac{C\left(\mathbf{x}_{1}, \mathbf{x}_{2}\right)}{\sigma\left(\mathbf{x}_{1}\right) \sigma\left(\mathbf{x}_{2}\right)}
$$

The random field is a Gaussian field if any vector $\left[Y\left(\mathbf{x}_{1}\right) \ldots Y\left(\mathbf{x}_{n}\right)\right]^{\mathrm{T}}$ is Gaussian. The Gaussian field is completely defined by its mean $\mu(\mathbf{x})$, variance $\sigma^{2}(\mathbf{x})$ and autocorrelation functions $\rho\left(\mathbf{x}_{1}, \mathbf{x}_{2}\right)$. If the random field is homogenous (or stationary for one-dimensional case), its covariance (and correlation) function will depend on the relative position of the pints $\mathbf{x}$ and $\mathbf{x}^{\prime}$ only, so we may write the covariance function as $C\left(\mathbf{x}, \mathbf{x}^{\prime}\right)=C\left(\mathbf{x}-\mathbf{x}^{\prime}\right)$. This paper only studies the homogenous random field.

The typical exponential and square exponential correlation functions are given as follows:

$$
\rho\left(\mathbf{x}, \mathbf{x}^{\prime}\right)=\exp \left(-\left\|\mathbf{x}-\mathbf{x}^{\prime}\right\| / a\right)
$$




$$
\rho\left(\mathbf{x}, \mathbf{x}^{\prime}\right)=\exp \left(-\left\|\mathbf{x}-\mathbf{x}^{\prime}\right\|^{2} / a^{2}\right)
$$

where $a$ is the correlation length.

The spectral representation of stationary random process can be stated as [25]

$$
Y(\mathbf{x}, \theta)=\int_{-\infty}^{\infty} e^{i \mathbf{x}^{\mathrm{T}} \mathbf{\omega}} d \mu(\boldsymbol{\omega}, \theta)
$$

where $d \mu$ is an orthogonal set function, also termed as orthogonal stochastic measure, defined on the $\sigma$-algebra $F$ of random events, and $\boldsymbol{\omega}$ is the wave number vector. This representation involves differentials of random functions so that it sets in an infinite dimensional space, which is not readily implemented by computational algorithms. The K-L expansion, which expands the random field $Y(\mathbf{x}, \theta)$ in the infinite dimensional space to a finite number of orthogonal random variables, is widely used to discretize the random field [26]. The random field $Y(\mathbf{x}, \theta)$ can be expanded to a Fourier-type series by the K-L expansion method, that is

$$
Y(\mathbf{x}, \theta)=\mu(\mathbf{x})+\sum_{i=1}^{\infty} \sqrt{\lambda_{i}} \mathrm{E}_{i}(\theta) f_{i}(\mathbf{x})
$$

where $\mu(\mathbf{x})$ is the mean of the random field, $\lambda_{i}$ is some constant, $\left\{\mathrm{E}_{i}(\theta)\right\}$ is a set of orthogonal random variables, and $\left\{f_{i}(\mathbf{x})\right\}$ is an orthonormal set of deterministic functions which form a complete orthogonal basis of $\mathcal{L}^{2}(\mathbf{D})$. Through the definition of covariance function $C\left(\mathbf{x}, \mathbf{x}^{\prime}\right)$ in Eq. (9), it is bounded, symmetric and positive definite, so it has the following spectral decomposition

$$
C\left(\mathbf{x}, \mathbf{x}^{\prime}\right)=\sum_{i=1}^{\infty} \lambda_{i} f_{i}(\mathbf{x}) f_{i}\left(\mathbf{x}^{\prime}\right)
$$

where $\lambda_{i}$ and $f_{i}(\mathbf{x})$ are the eigenvalue and the eigenvector of the covariance kernel, respectively. They are the solution to the following integral equation

$$
\int_{\mathbf{D}} C\left(\mathbf{x}, \mathbf{x}^{\prime}\right) f_{i}(\mathbf{x}) d(\mathbf{x})=\lambda_{i} f_{i}\left(\mathbf{x}^{\prime}\right)
$$

Due to non-accumulation of eigenvalues around a non-zero value, they can be sorted as a descending series converging to zero. We cannot use the infinite series to represent the random field, so the expansion is usually truncated by the first $m$ terms, that is 


$$
Y(\mathbf{x}, \theta) \approx \mu(\mathbf{x})+\sum_{i=1}^{m} \sqrt{\lambda_{i}} \mathrm{E}_{i}(\theta) f_{i}(\mathbf{x})
$$

It should be noted that $\left\{\mathrm{E}_{i}(\theta)\right\}$ is a set of independent standard Gaussian random variables if the random field is a Gaussian field. For sake the simplicity, only the Gaussian field is investigated in this study. After solving the Eq. (15), we can obtain the K-L expansion of a random field. Only few covariance functions have the closed-form solution for the Eq. (15), so the numerical method has to be used, which can be found in reference [25]. However, the orthogonal basis $\left\{f_{i}(\mathbf{x})\right\}$ obtained by the numerical method is no more optimal [26], thus another discretization method termed as expansion optimal linear estimation will be used, shown in the following subsection.

\subsection{EOLE method for discretizing the random field}

The expansion optimal linear estimation (EOLE) method was proposed in the reference [28]. Discretize the random field $Y(\mathbf{x}, \theta)$ to an approximated field $\hat{Y}(\mathbf{x}, \theta)$ by a linear function of nodal values $\mathbf{X}=$ $\left[Y\left(\mathbf{x}_{1}\right), \ldots Y\left(\mathbf{x}_{q}\right)\right]^{\mathrm{T}}$ as follows:

$$
\hat{Y}(\mathbf{x}, \theta)=A(\mathbf{x})+\mathbf{B}^{\mathrm{T}}(\mathbf{x}) \mathbf{X}
$$

where $A(\mathbf{x})$ is a function, $\mathbf{B}(\mathbf{x})=\left[B_{1}(\mathbf{x}), \ldots, B_{q}(\mathbf{x})\right]^{\mathrm{T}}$ is a function vector, and $q$ is the number of nodal points involved in the approximation. The functions $A(\mathbf{x})$ and $\mathbf{B}(\mathbf{x})$ are determined by minimizing the variance of the error in each point $\mathbf{x}$ subject to $\hat{Y}(\mathbf{x}, \theta)$ being an unbiased estimator of $Y(\mathbf{x}, \theta)$ in the mean. Thus, there is the following optimal problem:

$$
\begin{array}{ll}
\forall \mathbf{x} \in \mathbf{D}, \quad \min & \operatorname{Var}[Y(\mathbf{x}, \theta)-\hat{Y}(\mathbf{x}, \theta)] \\
& \text { s.t. } \quad \mathrm{E}[Y(\mathbf{x}, \theta)-\hat{Y}(\mathbf{x}, \theta)]=0
\end{array}
$$

Solving the above optimization problem, the functions $A(\mathbf{x})$ and $\mathbf{B}(\mathbf{x})$ can be determined as [26]

$$
A(\mathbf{x})=\mu(\mathbf{x})-\mathbf{B}^{\mathrm{T}}(\mathbf{x}) \mu_{\mathbf{x}}, \quad \mathbf{B}(\mathbf{x})=\mathbf{C}_{\mathbf{x x}}^{-1} \mathbf{C}_{\mathbf{x} \mathbf{x}}
$$

where $\mu(\mathbf{x})$ is the mean of nodal values $\mathbf{X}=\left[Y\left(\mathbf{x}_{1}\right), \ldots, Y\left(\mathbf{x}_{q}\right)\right]^{\mathrm{T}}, \mathbf{C}_{\mathbf{x} \mathbf{X}}$ denotes a vector with entry $C\left(\mathbf{x}, X_{i}\right)$, and $\mathbf{C}_{\mathbf{x x}}$ is the covariance matrix of nodal values with entry $\mathbf{C}_{\mathbf{x x}}(k, l)=C\left(\mathbf{x}_{k}, \mathbf{x}_{l}\right)$. Substituting the Eq. (19) into Eq. (17), the approximated field is expressed as 


$$
\hat{Y}(\mathbf{x}, \theta)=\mu(\mathbf{x})+\mathbf{C}_{\mathbf{x} \mathbf{x}}^{\mathrm{T}} \mathbf{C}_{\mathbf{x} \mathbf{x}}^{-1}\left(\mathbf{X}-\mu_{\mathbf{x}}\right)
$$

The spectral decomposition of the covariance matrix $\mathbf{C}_{\mathbf{X X}}$ of $\mathbf{X}$ is expressed by:

$$
\mathbf{X}(\theta) \approx \mu_{\mathbf{x}}+\sum_{i=1}^{q} \sqrt{\lambda_{i}} \mathrm{E}_{i}(\theta) \phi_{i}
$$

The $\left\{\mathrm{E}_{i}(\theta)\right\}(i=1, \ldots, q)$ is a set of independent standard Gaussian variables and $\left(\lambda_{i}, \phi_{i}\right)$ are the eigenvalues and eigenvectors of the covariance matrix $\mathbf{C}_{\mathbf{X X}}$, computed by solving the following eigenvalue problem

$$
\mathbf{C}_{\mathbf{X x}} \phi_{i}=\lambda_{i} \phi_{i}
$$

Substituting Eqs. (21) and (22) into Eq. (20), we can obtain the approximated field of EOLE

$$
\hat{Y}(\mathbf{x}, \theta)=\mu(\mathbf{x})+\mathbf{C}_{\mathbf{x} \mathbf{x}}^{\mathrm{T}} \mathbf{C}_{\mathbf{x x}}^{-1}\left(\sum_{i=1}^{q} \sqrt{\lambda_{i}} \mathrm{E}_{i}(\theta) \phi_{i}\right)=\mu(\mathbf{x})+\sum_{i=1}^{q} \frac{\mathrm{E}_{i}(\theta)}{\sqrt{\lambda_{i}}} \mathbf{C}_{\mathbf{x} \mathbf{x}}^{\mathrm{T}} \phi_{i}
$$

After sort the eigenvalues in descending order, the series in Eq. (23) can also be truncated by first $m$ terms. For the homogenous Gaussian field and considering the Eq. (10), the approximated field can be simplified as

$$
\hat{Y}(\mathbf{x}, \theta)=\mu+\sigma \sum_{i=1}^{m} \frac{\mathrm{E}_{i}(\theta)}{\sqrt{\lambda_{i}}} \boldsymbol{\rho}_{\mathbf{x} \mathbf{x}}^{\mathrm{T}} \phi_{i}
$$

where the vector $\boldsymbol{\rho}_{\mathbf{x x}}=\left[\rho\left(\mathbf{x}, \mathbf{x}_{1}\right), \ldots, \rho\left(\mathbf{x}, \mathbf{x}_{q}\right)\right]^{\mathrm{T}}$. The variance of the error for EOLE is:

$$
\operatorname{Var}[Y(\mathbf{x}, \theta)-\hat{Y}(\mathbf{x}, \theta)]=\sigma^{2}-\sum_{i=1}^{m} \frac{1}{\lambda_{i}}\left(\boldsymbol{\rho}_{\mathbf{x} \mathbf{x}}^{\mathrm{T}} \phi_{i}\right)^{2}
$$

The value of $m$ can be determined by make the relative variance error be lower than a small constant, e.g. $5 \%$ used in this study. Thus, the original random field has been approximated by a series governed by the $m$ independent standard normal variables $\left\{\mathrm{E}_{i}(\theta)\right\}(i=1, \ldots, m)$, which will be investigated in Section 4.

\section{Polynomial Chaos expansion for uncertainty system}

Since the random field has been discretized to $m$ independent standard normal variables $\mathrm{E}_{i}(\theta)$, the response of a system can be expressed by a function with respect these random variables $f(\mathbf{E}(\theta)), \mathbf{E} \in \mathrm{R}^{m}$, 
although the analytic expression of which is not known. This section uses the PC expansion to obtain the approximated analytic expression of the function and estimate its mean and covariance.

\subsection{PC expansion theory}

Considering the 1-dimensional case firstly, let $f(\mathrm{E})$ be a function of a random variable $\mathrm{E}$ whose probability distribution is $F_{\mathrm{E}}(\varepsilon)=P(\mathrm{E} \leq \varepsilon)$ and its support is $I_{\mathrm{E}}$. A generalized polynomial chaos approximation in a strong sense is $f_{n}(\varepsilon) \in \mathrm{P}_{n}(\mathrm{E})$, where $\mathrm{P}_{n}(\mathrm{E})$ is the space of polynomials of random variable $\mathrm{E}$ of degree up to $n \geq 0$, such that $\left\|f(\mathrm{E})-f_{n}(\mathrm{E})\right\| \rightarrow 0$ as $n \rightarrow \infty$, in a proper norm defined on $I_{\mathrm{E}}$ [46]. For any function $f(\mathrm{E})$ in the mean-square integrable space, its $n$ th-degree $\mathrm{PC}$ orthogonal projection is defined as

$$
f(\mathrm{E}) \approx \sum_{i=0}^{n} \hat{f}_{i} \Psi_{i}(\mathrm{E}), \hat{f}_{i}=\frac{1}{\gamma_{i}} \mathrm{E}\left[f(\mathrm{E}) \Psi_{i}(\mathrm{E})\right]
$$

This projection is the mean-square convergence. The $\hat{f}_{i}$ is the coefficients of the PC expansion, and $\Psi_{i}(\mathrm{E})$ denotes PC basic functions that are the orthogonal polynomial functions satisfying the following equation.

$$
\mathrm{E}\left[\Psi_{i}(\mathrm{E}) \Psi_{j}(\mathrm{E})\right]=\gamma_{i} \delta_{i j}, \gamma_{i}=\mathrm{E}\left[\Psi_{i}^{2}(\mathrm{E})\right], i, j=0,1, \ldots, n
$$

where $\gamma_{i}$ are the normalization factors, and $\delta_{i j}$ is the Kronecker delta function. For the continuous random variable E, its probability density function is denoted by $\tilde{\rho}(\varepsilon)$, so the orthogonality can be expressed by

$$
\mathrm{E}\left[\Psi_{i}(\mathrm{E}) \Psi_{j}(\mathrm{E})\right]=\int \Psi_{i}(\varepsilon) \Psi_{j}(\varepsilon) \tilde{\rho}(\varepsilon) d \varepsilon=\gamma_{i} \delta_{i j}
$$

Therefore, the $\left\{\Psi_{i}(\varepsilon)\right\}$ are orthogonal polynomials of $\varepsilon \in \mathrm{R}$ with the weight function $\tilde{\rho}(\varepsilon)$, which is the probability density function of the random variable E. The coefficients are computed by the following formula

$$
\hat{f}_{i}=\frac{1}{\gamma_{i}} \mathrm{E}\left[f(\mathrm{E}) \Psi_{i}(\mathrm{E})\right]=\frac{1}{\gamma_{i}} \int f(\varepsilon) \Psi_{i}(\varepsilon) \tilde{\rho}(\varepsilon) d \varepsilon
$$

For the probability distribution function of the standard normal random variable E, its probability density function is given as $\tilde{\rho}(\varepsilon)=\frac{1}{\sqrt{2 \pi}} e^{-\varepsilon^{2} / 2}$, and the corresponding orthogonal polynomials are the Hermite polynomials, which are expressed by 


$$
H_{0}(\mathrm{E})=1 ; H_{1}(\mathrm{E})=\mathrm{E} ; H_{i}(\mathrm{E})=\mathrm{E} H_{i-1}(\mathrm{E})-H_{i-2}(\mathrm{E}), i=2,3 \ldots
$$

Therefore, the Hermite polynomials will be used as the basis of PC expansion for normal random variable, and the corresponding normalization factor is $\gamma_{i}=i$, which can be obtained through Eq. (27).

For the $m$-dimensional case, the bases are defined as the product of the each univariate Hermite polynomials, i.e.

$$
\Psi_{\mathbf{i}}(\mathbf{E})=\prod_{k=1}^{m} H_{i_{k}}\left(\mathrm{E}_{k}\right), i_{k}=0,1, \ldots
$$

where the subscript $\mathbf{i}$ is a $m$-dimensional multi-index. As a result, the $n$ th-degree PC expansion of function $f(\mathbf{E}), \mathbf{E} \in \mathbb{R}^{m}$, is expressed as the following equation

$$
f(\mathbf{E}) \approx \sum_{\mid \mathrm{i}_{0} \leq n} \hat{f}_{\mathbf{i}} \Psi_{\mathbf{i}}(\mathbf{E}), \hat{f}_{\mathbf{i}}=\frac{1}{\gamma_{\mathbf{i}}} \mathrm{E}\left[f(\mathbf{E}) \Psi_{\mathbf{i}}(\mathbf{E})\right]
$$

where $|\mathbf{i}|_{0}=\max i_{k}, 1 \leq k \leq m$, and the number of coefficients is $(n+1)^{m}$.

After the PC expansion, based on the orthogonality, the mean and variance of the function $f(\mathbf{E})$ can be computed by the following equations

$$
\begin{gathered}
\mu_{f}=\mathbb{E}[f(\mathbf{E})] \approx \int \sum_{\mid \mathrm{i}_{0} \leq n} \hat{f}_{\mathbf{i}} \Psi_{\mathbf{i}}(\boldsymbol{\varepsilon}) d \boldsymbol{\varepsilon}=\hat{f}_{\mathbf{0}} \\
\sigma_{f}^{2}=\mathrm{E}\left[\left(f(\mathbf{E})-\mu_{f}\right)^{2}\right] \approx \sum_{0<\mathrm{i}_{0} \leq n} \gamma_{\mathbf{i}} \hat{f}_{\mathbf{i}}^{2}
\end{gathered}
$$

It can be found that after the coefficients of PC expansion are determined, the mean and variance of the function $f(\mathbf{E})$ can be obtained conveniently. If the output of a function is a vector, that is $\mathbf{f}(\mathbf{E}): \mathrm{R}^{m} \rightarrow \mathbb{R}^{k}$, we can compute the covariance between each output as

$$
\sigma_{l j}=\mathrm{E}\left[\left(f_{l}(\mathbf{E})-\mu_{f_{l}}\right)\left(f_{j}(\mathbf{E})-\mu_{f_{j}}\right)\right] \approx \sum_{0<\mathbf{i}_{0} \leq n} \gamma_{\mathbf{i}} \hat{f}_{l, \mathbf{i}} \hat{f}_{j, \mathbf{i}}, l, j=1, \ldots, k
$$

\subsection{Numerical implementation method}

The key of PC expansion is to compute the coefficients that are determined by the Eq. (29). Since the analytical expression of function $f(\mathbf{E})$ is not known, we have to use the numerical quadrature formula to compute it. Here we use the interpolation integral formula to calculate the integration, defined as 


$$
\int_{a}^{b} \rho(x) f(x) d x \approx \sum_{i=1}^{k} w_{i} f\left(x_{i}\right)
$$

where the $k$ is the order of the interpolation integral formula, $\rho(x)$ denotes the weight function, $w_{i}$ is the integral coefficient, and $x_{\mathrm{i}}$ denotes the interpolation points. When the interpolation points are the roots of $k$ th-order orthogonal polynomial with weight function $\rho(x)$, the algebraic precision order of this integral formula will be $2 k-1$, which is the Gaussian quadrature formula. When the weight function is $\rho(\varepsilon)=\tilde{\rho}(\varepsilon)$, the corresponding orthogonal polynomials will be the Hermite polynomials. Therefore, the Eq. (29) can be computed by using the following Gaussian-Hermite quadrature formula [47]

$$
\hat{f}_{i}=\frac{1}{\gamma_{i}} \int_{-\infty}^{\infty} \frac{1}{\sqrt{2 \pi}} f(\varepsilon) H_{i}(\varepsilon) e^{-\varepsilon^{2} / 2} d \varepsilon \approx \sum_{j=1}^{k} \frac{1}{\gamma_{i}} w_{j} f\left(\varepsilon_{j}\right) H_{i}\left(\varepsilon_{j}\right), w_{j}=\frac{k !}{k^{2} H_{k-1}^{2}\left(\varepsilon_{j}\right)}
$$

where the $\varepsilon_{j}$ is the roots of $k$ th-order univariate Hermite polynomials. To keep the numerical accuracy, the order $k$ is required to be larger than the order of PC expansion $n$, so we set $k=n+1$ to save the computational cost.

Writing the Eq. (37) in the format of matrix, the coefficient vector can be expressed by following formula

$$
\hat{\mathbf{f}}=\boldsymbol{\Gamma}^{-1} \mathbf{H}^{\mathrm{T}}(\boldsymbol{\varepsilon}) \mathbf{\Omega} \mathbf{f}(\boldsymbol{\varepsilon})
$$

where the $\hat{\mathbf{f}}=\left[\hat{f}_{0}, \hat{f}_{1}, \ldots \hat{f}_{n}\right]^{\mathrm{T}}$ denotes the coefficient vector, $\boldsymbol{\Gamma}$ is a diagonal matrix with entry $\Gamma_{i i}=\gamma_{i}$ (here the subscript start from 0$), \mathbf{H}(\boldsymbol{\varepsilon})=\left[H_{0}(\boldsymbol{\varepsilon}), \ldots H_{n}(\boldsymbol{\varepsilon})\right]$ is the transform matrix at the interpolation points $\boldsymbol{\varepsilon}=\left[\varepsilon 1, \ldots \varepsilon_{n+1}\right]^{\mathrm{T}}, \boldsymbol{\Omega}$ denotes the diagonal weighting matrix with entry $\Omega_{i i}=w_{i}$, and $\mathbf{f}(\boldsymbol{\varepsilon})=\left[f(\varepsilon 1), \ldots f\left(\varepsilon_{n+1}\right)\right]^{\mathrm{T}}$ is the vector of function value at the interpolation points. Besides using the quadrature formula, we can also use the collocation method which is realized by the least square method to compute the coefficients, that is

$$
\hat{\mathbf{f}}=\left(\mathbf{H}^{\mathrm{T}}(\boldsymbol{\varepsilon}) \mathbf{H}(\boldsymbol{\varepsilon})\right)^{-1} \mathbf{H}^{\mathrm{T}}(\boldsymbol{\varepsilon}) \mathbf{f}(\boldsymbol{\varepsilon})
$$

Theorem 1: When computing the coefficients of PC expansion, the Gaussian quadrature formula Eq. (38) and the least square method Eq. (39) will give the same result, if both the two methods use the roots of corresponding orthogonal polynomials as the interpolation points or collocation points.

Proof: We only need to prove that the right side of the Eq. (38) and (39) are equivalent, that is 


$$
\boldsymbol{\Gamma}^{-1} \mathbf{H}^{\mathrm{T}}(\boldsymbol{\varepsilon}) \boldsymbol{\Omega} \mathbf{f}(\boldsymbol{\varepsilon})=\left(\mathbf{H}^{\mathrm{T}}(\boldsymbol{\varepsilon}) \mathbf{H}(\boldsymbol{\varepsilon})\right)^{-1} \mathbf{H}^{\mathrm{T}}(\boldsymbol{\varepsilon}) \mathbf{f}(\boldsymbol{\varepsilon})
$$

The previous equation indicates the following equation

$$
\boldsymbol{\Gamma}^{-1} \mathbf{H}^{\mathrm{T}}(\boldsymbol{\varepsilon}) \boldsymbol{\Omega}=\left(\mathbf{H}^{\mathrm{T}}(\boldsymbol{\varepsilon}) \mathbf{H}(\boldsymbol{\varepsilon})\right)^{-1} \mathbf{H}^{\mathrm{T}}(\boldsymbol{\varepsilon})
$$

Postmultiplying $\mathbf{H}(\boldsymbol{\varepsilon})$ to the two sides, the previous equation can be transformed to

$$
\boldsymbol{\Gamma}^{-1} \mathbf{H}^{\mathrm{T}}(\boldsymbol{\varepsilon}) \boldsymbol{\Omega} \mathbf{H}(\boldsymbol{\varepsilon})=\left(\mathbf{H}^{\mathrm{T}}(\boldsymbol{\varepsilon}) \mathbf{H}(\boldsymbol{\varepsilon})\right)^{-1} \mathbf{H}^{\mathrm{T}}(\boldsymbol{\varepsilon}) \mathbf{H}(\boldsymbol{\varepsilon})=\mathbf{I}
$$

where $I$ is the unit matrix with size $n+1$ by $n+1$. To validate the Eq. (42), use the ( $n+1)$ th-order Gaussian-Hermite quadrature formula to compute the following integral.

$$
\int \tilde{\rho}(\varepsilon) H_{i}(\varepsilon) H_{j}(\varepsilon) d \varepsilon=\sum_{k=1}^{n+1} w_{k} H_{i}\left(\varepsilon_{k}\right) H_{j}\left(\varepsilon_{k}\right), 0 \leq i, j \leq n
$$

where $\varepsilon k$ denotes the roots of Hermite polynomials with order $n+1$. Since the algebraic precision order of the Gaussian quadrature formula is $2 n+1$, as well as the order of $H_{i}$ and $H_{j}$ is no more than $n$, the two sides of the previous formula are exactly equivalent. Considering the orthogonality of Hermite polynomials (Eq. (28)), we have

$$
\sum_{k=1}^{n+1} w_{k} H_{i}\left(\varepsilon_{k}\right) H_{j}\left(\varepsilon_{k}\right)=\gamma_{i} \delta_{i j}, \quad 0 \leq i, j \leq n
$$

Rewrite the Eq. (44) as the format of matrix, which lead to the following equation

$$
\mathbf{H}^{\mathrm{T}}(\boldsymbol{\varepsilon}) \boldsymbol{\Omega H}(\boldsymbol{\varepsilon})=\boldsymbol{\Gamma}
$$

Premultiplying $\Gamma^{-1}$ to the two sides of Eq. (45), we can obtain the Eq. (42), so the theorem is proved.

For the $m$-dimensional case, we can still use the least square method to compute the coefficients, changing the univariate Hermite polynomials to the multi-dimensional Hermite polynomials, i.e.

$$
\hat{\mathbf{f}}=\left(\boldsymbol{\Psi}^{\mathrm{T}}(\tilde{\boldsymbol{\varepsilon}}) \boldsymbol{\Psi}(\tilde{\boldsymbol{\varepsilon}})\right)^{-1} \boldsymbol{\Psi}^{\mathrm{T}}(\tilde{\boldsymbol{\varepsilon}}) \mathbf{f}(\tilde{\boldsymbol{\varepsilon}})
$$

where the coefficient is expressed by a vector $\hat{\mathbf{f}}=\left[\hat{f}_{0}, \ldots \hat{f}_{\mathbf{n}}\right]^{\mathrm{T}}$ with $(n+1)^{m}$ rows, $\mathbf{f}(\tilde{\boldsymbol{\varepsilon}})$ are function values at the given collocation points $\tilde{\boldsymbol{\varepsilon}}$, and $\boldsymbol{\Psi}(\tilde{\boldsymbol{\varepsilon}})$ denotes the transform matrix with size $(n+1)^{m}$ by $(n+1)^{m}$, 
defined by

$$
\Psi(\tilde{\boldsymbol{\varepsilon}})=\left[\begin{array}{lll}
\Psi_{0}(\tilde{\boldsymbol{\varepsilon}}), & \cdots & \Psi_{\mathbf{n}}(\tilde{\boldsymbol{\varepsilon}})
\end{array}\right]
$$

The collocation points $\tilde{\boldsymbol{\varepsilon}}$ are the tensor product of the roots of univariate Hermite polynomials with order $n+1$ in $m$ dimensional space, that is

$$
\tilde{\boldsymbol{\varepsilon}}=\boldsymbol{\varepsilon}_{1} \otimes \cdots \otimes \boldsymbol{\varepsilon}_{m}, \boldsymbol{\varepsilon}_{i}=\left[\varepsilon_{1}, \cdots \varepsilon_{n+1}\right]^{\mathrm{T}}, i=1,2, \ldots, m
$$

It can be found that this procedure is same as constructing a response surface model. The only difference is that the basis are Hermite polynomials rather than the monomials used in traditional response surface model, so this method is also termed as stochastic response surface method (SRSM) [48].

\section{Computational strategy for solving the system}

In the previous section, we have solved the problem how to estimate the mean and variance of a function with random variables, but it requires computing the function values $\mathbf{f}(\tilde{\boldsymbol{\varepsilon}})$ at some collocation points. For this study, computing the function values $\mathbf{f}(\tilde{\boldsymbol{\varepsilon}})$ need to solve the flexible multibody system, which will be provided in this section.

Based on the ANCF, the assembly of the elements can be implemented by traditional finite element method. The element nodal coordinates e can be transformed into the flexible multibody system generalized coordinates q. The equations of motion for a constrained flexible multibody system can be expressed in a compact form of differential algebraic equations (DAEs) as [5]

$$
\left\{\begin{array}{l}
\mathbf{M} \ddot{\mathbf{q}}+\boldsymbol{\Phi}_{\mathbf{q}}^{\mathrm{T}} \lambda+\mathbf{F}(\mathbf{q})=\mathbf{Q}(\mathbf{q}) \\
\boldsymbol{\Phi}(\mathbf{q}, t)=\mathbf{0}
\end{array}\right.
$$

where $\mathbf{M}$ is the system mass matrix, $\mathbf{\Phi}(\mathbf{q}, t)$ is the vector that contains the system constraint equations corresponding to ideal or perfect joints, $t$ represents the time, $\boldsymbol{\Phi}_{\mathbf{q}}$ is the derivative matrix of constraint equations with respect to the system generalized coordinates $\mathbf{q}, \lambda$ is the Lagrangian multipliers associated with the constraints, $\mathbf{Q}(\mathbf{q})$ is the system external generalized forces, e.g. the gravity force in this study, and $\mathbf{F}(\mathbf{q})$ denotes the system elastic force vector that can be computed by Eq. (7). A variety of numerical methods can be used to solve the DAEs shown in Eq. (49), which can be found in the literature [49]. The generalized- $\boldsymbol{\alpha}$ method [50] will be used in this paper, since it has a good trade-off between the numerical 
accuracy at low-frequency and numerical damping at high-frequency. More detailed iteration procedure of the generalized- $\boldsymbol{\alpha}$ algorithm can be found in the reference [50].

The generalized- $\boldsymbol{\alpha}$ method only solve the traditional deterministic flexible multibody system. When the uncertainties of material properties are considered, we do not need to modify the solver itself, but only add a pre-process and a post-process.

\subsection{Pre-process for solving uncertain flexible multibody system}

In this study, the Young's modulus and Poisson's ratio are considered as Gaussian random field. Using the EOLE discretization method shown in Section 3, the Young's modulus $G$ and Poisson's ratio $\kappa$ can be approximated by

$$
G(\mathbf{x}, \theta)=\mu_{G}+\sigma_{G} \sum_{i=1}^{m} \frac{\mathrm{E}_{i}(\theta)}{\sqrt{\lambda_{i}}} \boldsymbol{\rho}_{\mathbf{x} \mathbf{x}}^{\mathrm{T}} \phi_{i}, \kappa(\mathbf{x}, \theta)=\mu_{\kappa}+\sigma_{\kappa} \sum_{i=1}^{m} \frac{\mathrm{E}_{i}(\theta)}{\sqrt{\lambda_{i}}} \boldsymbol{\rho}_{\mathbf{x} \mathbf{x}}^{\mathrm{T}} \phi_{i}
$$

Here the $G$ and $\kappa$ are changed in the space domain, so the expression of the constant stiffness matrix Eq. (6) and invariant matrix Eq. (8) will be modified as

$$
\begin{aligned}
\mathbf{K}_{1}^{2 D} & =-\int_{V}(\kappa(\mathbf{x}, \theta)+G(\mathbf{x}, \theta)) \sum_{\alpha=1}^{2} \mathbf{S}_{, \alpha}^{\mathrm{T}} \mathbf{S}_{, \alpha} d V, \mathbf{K}_{1}^{3 D}=-\int_{V} \frac{3 \kappa(\mathbf{x}, \theta)+2 G(\mathbf{x}, \theta)}{2} \sum_{\alpha=1}^{3} \mathbf{S}_{, \alpha}^{\mathrm{T}} \mathbf{S}_{, \alpha} d V \\
\mathbf{C}_{\mathbf{K}_{2}}^{i j}= & \sum_{\alpha=1}^{N} \int_{V} \frac{\kappa(\mathbf{x}, \theta)+2 G(\mathbf{x}, \theta)}{2}\left(\mathbf{S}_{, \alpha}^{\mathrm{T}} \mathbf{S}_{, \alpha}\right)_{i}^{T}\left(\mathbf{S}_{, \alpha}^{\mathrm{T}} \mathbf{S}_{, \alpha}\right)_{j} d V \\
& +\sum_{\alpha=1}^{N} \sum_{\beta=1, \beta \neq \alpha}^{N} \int_{V} \frac{\kappa(\mathbf{x}, \theta)}{2}\left(\mathbf{S}_{, \alpha}^{\mathrm{T}} \mathbf{S}_{, \alpha}\right)_{i}^{T}\left(\mathbf{S}_{, \beta}^{\mathrm{T}} \mathbf{S}_{, \beta}\right)_{j} d V+\sum_{\alpha=1}^{N} \sum_{\beta=1, \beta \neq \alpha}^{N} \int_{V} G(\mathbf{x}, \theta)\left(\mathbf{S}_{, \alpha}^{\mathrm{T}} \mathbf{S}_{, \beta}\right)_{i}^{T}\left(\mathbf{S}_{, \alpha}^{\mathrm{T}} \mathbf{S}_{, \beta}\right)_{j} d V
\end{aligned}
$$

Since the two matrix are depend on the random variables $\mathbf{E}(\theta)$, the elastic forces are also depend on them, so the DAEs of the system will be modified as

$$
\left\{\begin{array}{l}
\mathbf{M} \ddot{\mathbf{q}}+\boldsymbol{\Phi}_{\mathbf{q}}^{\mathrm{T}} \lambda+\mathbf{F}(\mathbf{q}, \mathbf{E}(\theta))=\mathbf{Q}(\mathbf{q}) \\
\mathbf{\Phi}(\mathbf{q}, t)=\mathbf{0}
\end{array}\right.
$$

Considering the generalized coordinates $\mathbf{q}(\mathbf{E})$ as the function $f(\mathbf{E})$ in Section 4.2 and using the PC expansion method to solve this system, we just need to solve the previous DAEs by fixing the value random variable $\mathbf{E}(\theta)$ at the given collocation points $\tilde{\boldsymbol{\varepsilon}}$, which has the same procedure of solving traditional deterministic DAEs. After we obtain the generalized coordinates at the given collocation points, we can use the Eq. (46) to compute the coefficients of PC expansion. 


\subsection{Post-process for represent the displacement}

We can obtain the mean and variance of the displacement field by using Eq. (33) and Eq. (34). On the other hand, the displacements of each node at different direction are not independent, so the covariance should also be used describe the relationship. To show the uncertainty extent of displacement in each direction, a post-process is added. Considering the displacement field in global coordinate system of two-dimensional element, its mean and covariance can be expressed by

$$
\mu_{\mathbf{r}}=\left[\begin{array}{ll}
\mu_{r_{1}} & \mu_{r_{2}}
\end{array}\right]^{\mathrm{T}}, \operatorname{Cov}\left(r_{1}, r_{2}\right)=\left[\begin{array}{cc}
\sigma_{r_{1}}^{2} & \sigma_{r_{1} r_{2}} \\
\sigma_{r_{1} r_{2}} & \sigma_{r_{2}}^{2}
\end{array}\right]
$$

Use the eigenvalues analysis to transform the covariance matrix to a diagonal matrix, i.e. solving the following eigenvalues problem

$$
\left[\begin{array}{ll}
\tilde{\sigma}_{1}^{2} & \\
& \tilde{\sigma}_{2}^{2}
\end{array}\right]\left[\begin{array}{ll}
\varphi_{11} & \varphi_{12} \\
\varphi_{21} & \varphi_{22}
\end{array}\right]=\left[\begin{array}{cc}
\sigma_{r_{1}}^{2} & \sigma_{r_{1} r_{2}} \\
\sigma_{r_{1} r_{2}} & \sigma_{r_{2}}^{2}
\end{array}\right]\left[\begin{array}{ll}
\varphi_{11} & \varphi_{12} \\
\varphi_{21} & \varphi_{22}
\end{array}\right]
$$

where the eigenvalues $\tilde{\sigma}_{i}$ denotes the normalized standard deviation, and the eigenvectors determine the direction of the normalized standard deviation.

Therefore, when considering the uncertainty, the displacement of a node in one standard deviation can be plot by an ellipse, shown as Fig. 3. Similarly, the displacement of a node for the three-dimensional element can be plot as an ellipsoid.
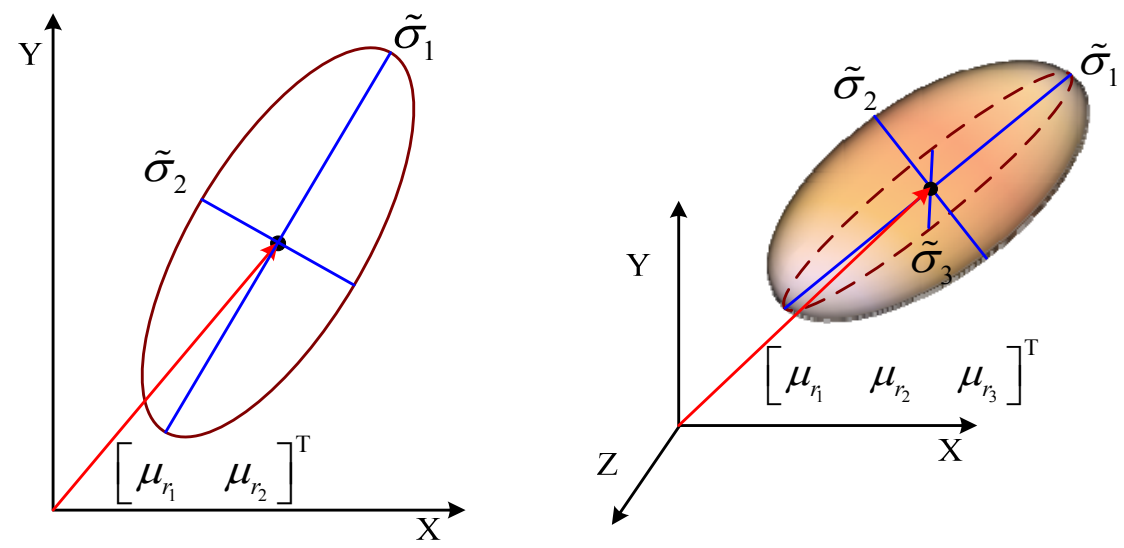

Figure.3 Plot of nodal displacement under uncertainty

The computation procedure of the flexible multibody system with uncertain material properties can be 
summarized as the following flowchart. It can be found that the proposed method mainly contains four steps: (1) discretize the random field to several independent normal random variables; (2) determine the collocation points of the random variables; (3) solve the DAEs using the generalized- $\alpha$ method at the given collocation points and then compute the coefficients of PC expansion; (4) compute the mean, covariance matrix, and normalized variance of the displacement of each node, as well as plot the ellipse and ellipsoid of the nodal displacement.



Figure. 4 Flowchart for solving flexible multibody system with uncertain material properties

\section{Numerical examples}

\subsection{Single plane pendulum with uncertain Poisson's ratio}

Considering a uniform distributed gravity force is applied to a single plane pendulum with $1 \mathrm{~m}$ length and rectangle cross-section $(0.05 \mathrm{~m}$ height and $0.01 \mathrm{~m}$ width $)$. The left end of the bar is connected by a revolute joint. The initial configuration of the single pendulum is shown as Fig. 5. 


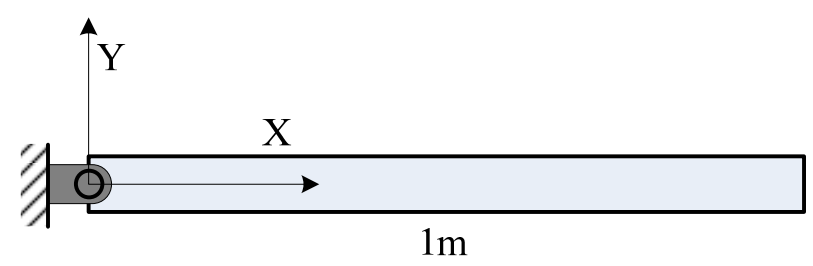

Figure. 5 The schematic of single plane pendulum

The gravity acceleration is $9.81 \mathrm{~m} / \mathrm{s}^{2}$ to the negative $\mathrm{Y}$ direction. The Young's modulus is $2 \times 10^{7} \mathrm{~Pa}$. The Poisson's ratio of the material is considered as a Gaussian random field along the $\mathrm{X}$ direction, and the correlation function is given by the following formula.

$$
\rho\left(X, X^{\prime}\right)=\exp \left(-\left\|X-X^{\prime}\right\|^{2} / 2^{2}\right)
$$

Here the correlation length is $2 \mathrm{~m}$. The mean of the Poisson's ratio is set as 0.3 , and the standard deviation is $5 \%$ of mean, i.e. 0.015 . Use the EOLE method to discretize the random field, truncated by 2 terms, which means the random field is discretized to two normal random variables. The error of variance after the discretization is shown in Fig. 6, which indicates that the maximum error is about $0.0032<5 \%$ required by this study.

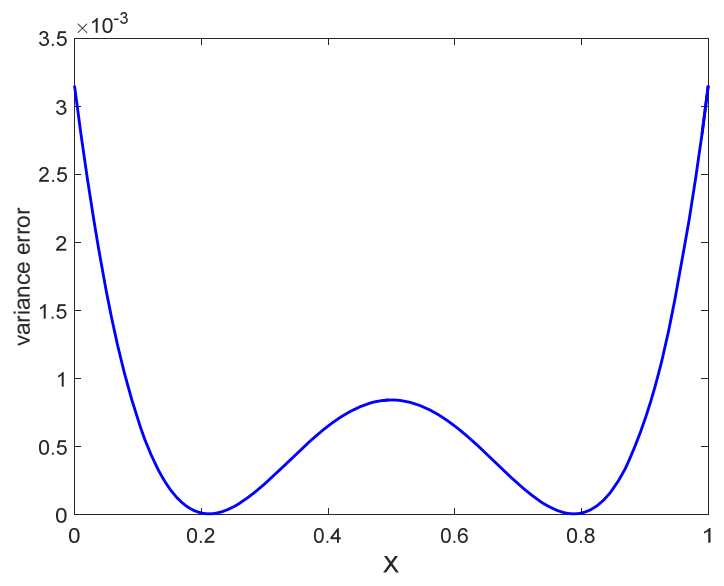

Figure. 6 Variance error along the $\mathrm{X}$ axis

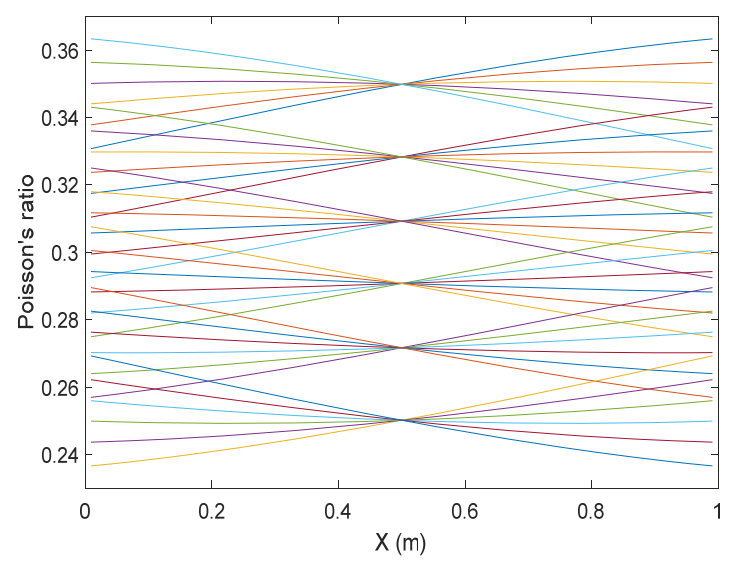

Figure. 7 Realizations at 36 collocation points

Use the 5th-order Hermite series to implement the PC expansion, so it requires at least 6 collocation points that are roots of the 6th-order Hermite polynomial, i.e. $\varepsilon=[-3.3243,-1.8892,-0.6167,0.6167$, $1.8892,3.3243]^{\mathrm{T}}$ for each random variable. Considering the tensor product operation of two random variables, there will be $6^{2}=36$ collocation points. Substituting the collocation points into the Eq. (24), 
we can obtain the corresponding 36 realizations along the length direction at these collocation points, shown in Fig. 7.

Five ANCF-based planar beam elements are used to discretize the pendulum. The configuration of the single pendulum changing from $0 \mathrm{~s}$ to $2 \mathrm{~s}$ is shown in Fig. 8. The legend "DET" denotes the deterministic case that the Poisson's ratio is considered as the mean value 0.3 , while the "PC" denotes the mean solution obtained by the PC expansion considering the uncertainty, and the 'STD-ELLIPSE' is the ellipse plot of normalized standard deviation for the nodal displacement. It can be found that there is an obvious difference between the solution of deterministic case and the uncertain case. Especially with time increasing, the difference becomes larger. The ellipse indicates the standard deviation of the displacement, which becomes larger with time increasing. Therefore, the uncertainty of Poisson's ratio will produce a large uncertainty of nodal displacement when the time increases.



Figure. 8(a) Configuration of single pendulum between 0 s to $2 \mathrm{~s}$ 


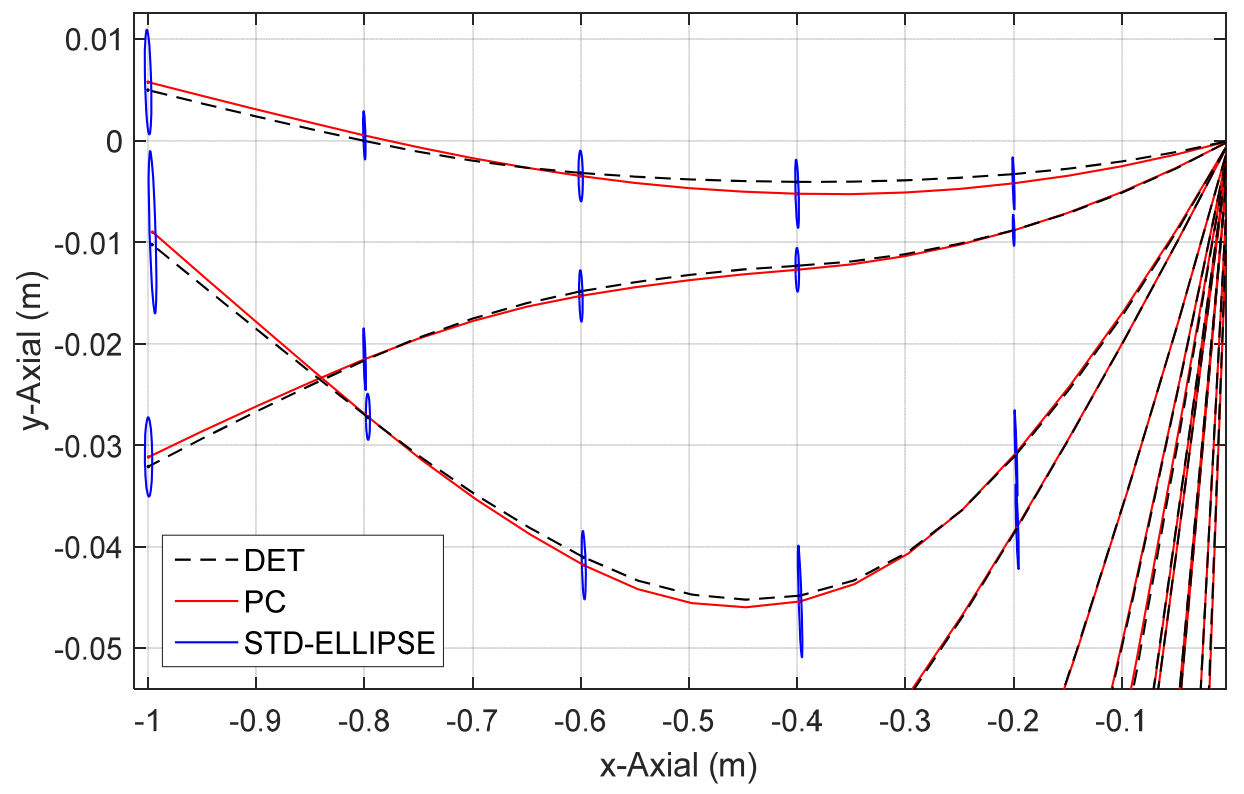

Figure. 8(b) Scaled view of Fig. 8(a)

To validate the PC expansion, the Monte Carlo method with 1000 sampling points is employed to give a reference solution. Figure 9 shows the 1000 realizations of the Poisson's ratio produced by the Monte Carlo method.

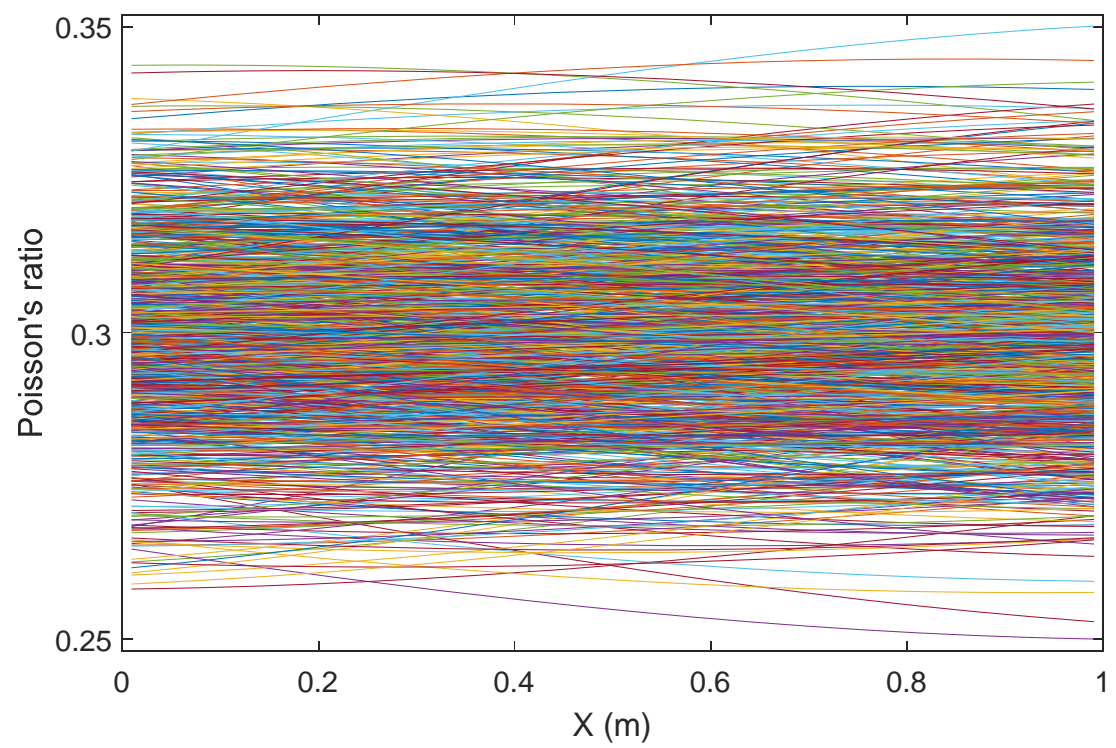

Figure. 9 The 1000 realizations used in Monte Carlo method

The mean displacement of the pendulum's end node is shown in Fig. 10. It is noted that the mean displacement of the end node obtained by the PC expansion and Monte Carlo simulation are coincident, 
so the accuracy of the PC expansion for computing the mean displacement is quite high.

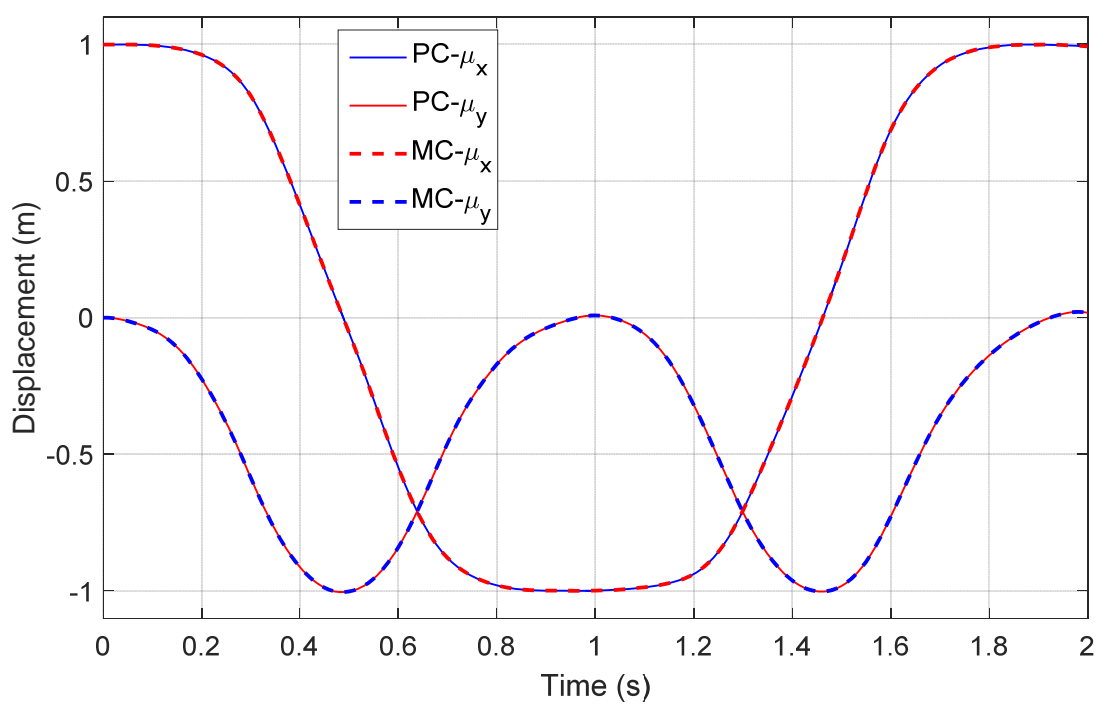

Figure. 10 Mean displacement of the end node

Figure 11 shows the covariance of the displacement of the end node change with the time. The magnitude of covariance increases with time increasing, which indicates that the uncertainty extent becomes larger and larger when time increases. On the other hand, the curves obtained by the PC expansion match the curves of Monte Carlo simulation in the whole time space, so the PC expansion can also estimate the covariance in high accuracy.

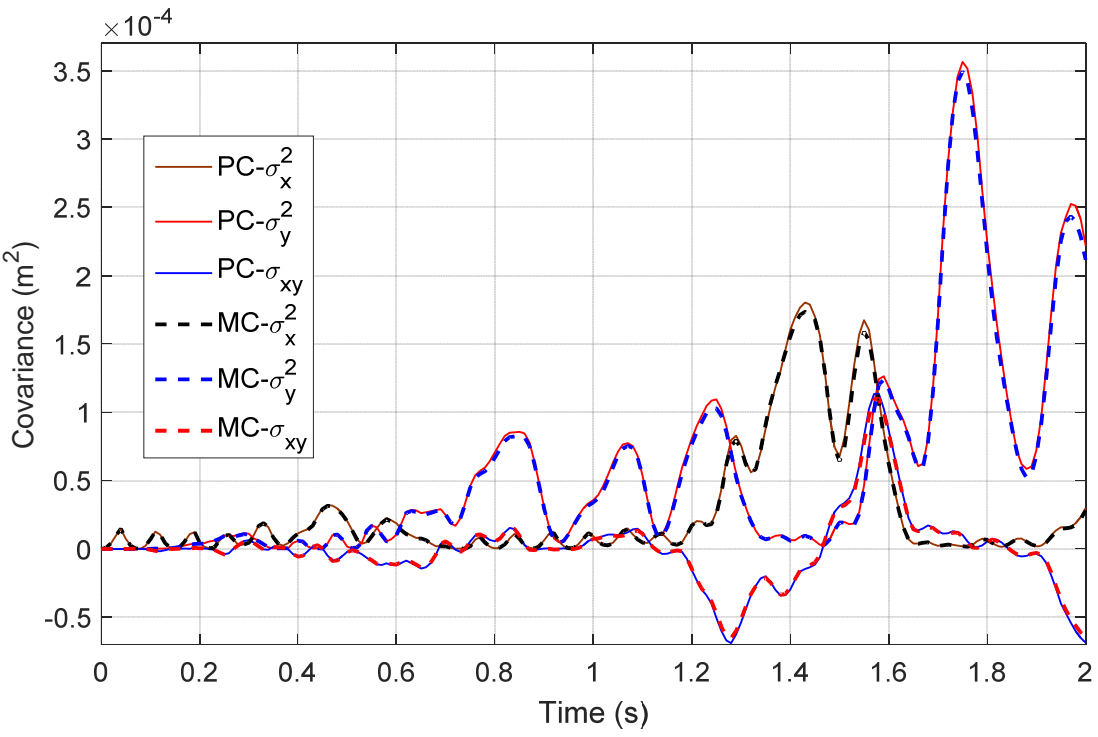

Figure. 11 Displacement covariance of the end node

Figure 12 shows the normalized standard deviation of displacement of the end node, where $\sigma_{1}$ is the long 
axis of the ellipse in Fig. 7 and $\sigma_{2}$ is the short axis of the ellipse. With time increasing, the normalized standard deviations tend to become larger, which also demonstrates that the uncertainty extent will increase with the time. The magnitude of the long axis increases faster than the short axis, which means that there are strong correlation between the variance in $\mathrm{X}$ and $\mathrm{Y}$ direction. The $\mathrm{PC}$ expansion still gives quite similar results to the Monte Carlo simulation, which further indicates the accuracy of the PC expansion. It should be noted that the efficiency of PC expansion is much higher than the Monte Carlo simulation, since the $\mathrm{PC}$ expansion only runs the deterministic model 36 times but the Monte Carlo method uses 1000 simulations.



Figure. 12 Normalized standard deviation of end node displacement

\subsection{Double space pendulum with uncertain Young's modulus}

The initial configuration of a double space pendulum is shown in Fig. 13.

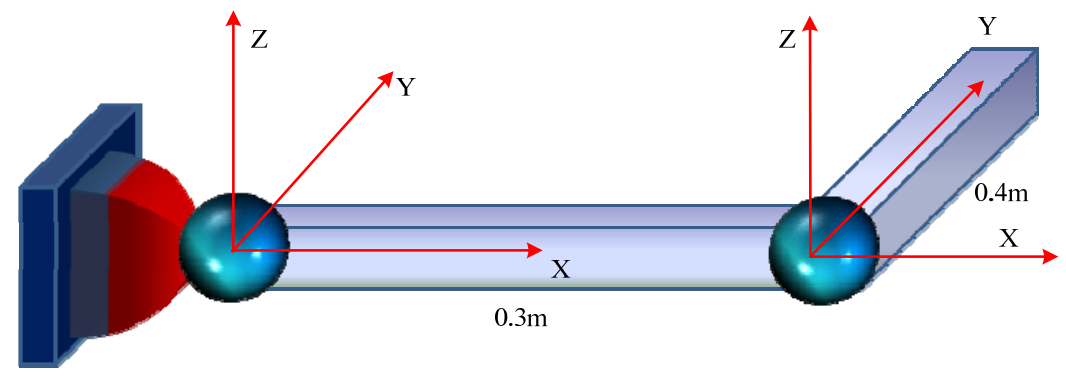

Figure. 13 The initial configuration of the double space pendulum

There are two sphere joints located between the base and the first pendulum, the first pendulum and the 
second pendulum, respectively. The length of the first pendulum is $0.3 \mathrm{~m}$, while the second pendulum is $0.4 \mathrm{~m}$ length. The cross-section of both two pendulums is a square with edge length $0.01 \mathrm{~m}$. The gravity force of the two pendulums is uniformly distributed with gravity acceleration $9.81 \mathrm{~m} / \mathrm{s}^{2}$ in negative $\mathrm{Z}$ direction.

The two pendulums are made by the same material with Poisson's ratio 0.3 . The Young's modulus of the two pendulums is considered as a Gaussian random field along the length direction, and the correlation function is given by the following formula.

$$
\rho\left(X, X^{\prime}\right)=\exp \left(-\left\|X-X^{\prime}\right\|^{2} / 1^{2}\right)
$$

where the correlation length is $1 \mathrm{~m}$. The mean of Young's modulus is $2 \times 10^{7} \mathrm{~Pa}$, while its standard deviation is $10 \%$ of mean, i.e. $2 \times 10^{6} \mathrm{~Pa}$.

Using the EOLE method to discretize the random field, the first pendulum is truncated by 1 term while the second pendulum is truncated by 2 terms. Therefore, the first pendulum is controlled by one normal random variable, and the second pendulum is controlled by two independent normal random variables. The variance error of the discretization is shown in Fig. 14. Since the maximum error of variance is smaller than $5 \%$, this truncation can be accepted.

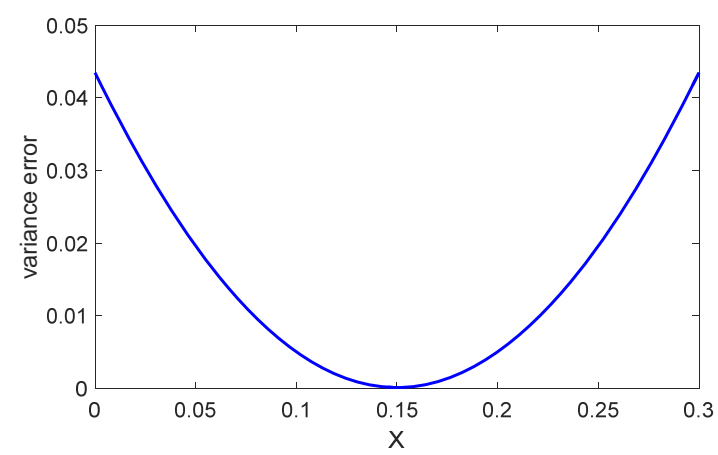

(a) The first pendulum

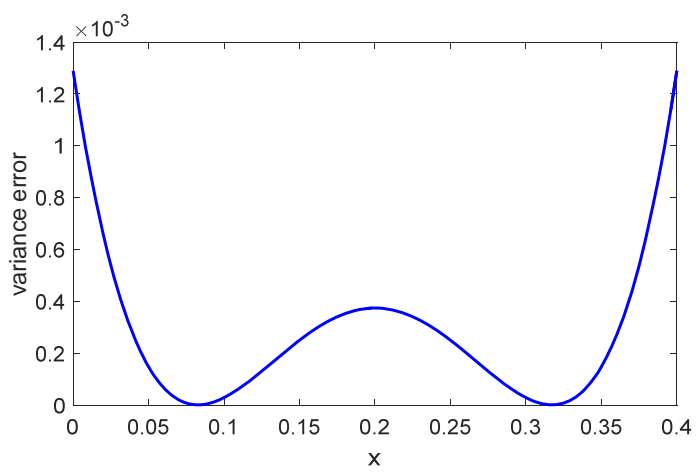

(b) The second pendulum

Figure. 14 Variance error along the length direction

Considering the second order PC expansion, there will be 3 collocation points that are $\varepsilon=[-1.7321,0$, $1.7321]^{\mathrm{T}}$ in each dimensional random variable. Therefore, there are 3 realizations of Young's modulus for the first pendulum and 9 realizations for the second pendulum, shown as the Fig. 15. It should be noted 
that for the whole system there are $3 \times 9=27$ collocation points, which means 27 runs of the deterministic model are required in the PC expansion.

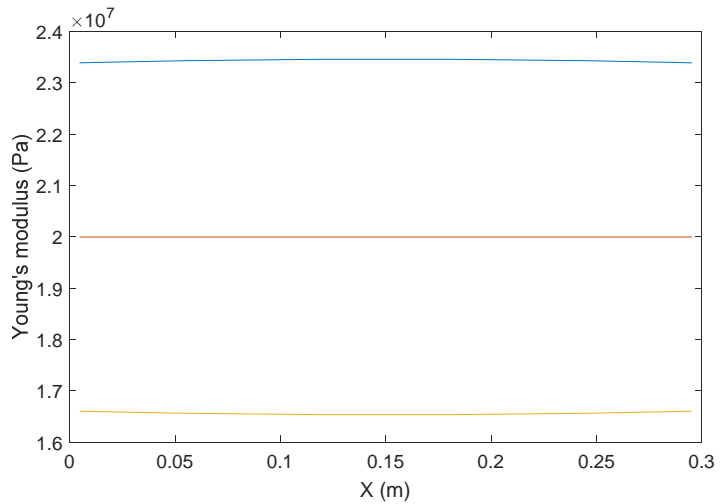

(a) The first pendulum

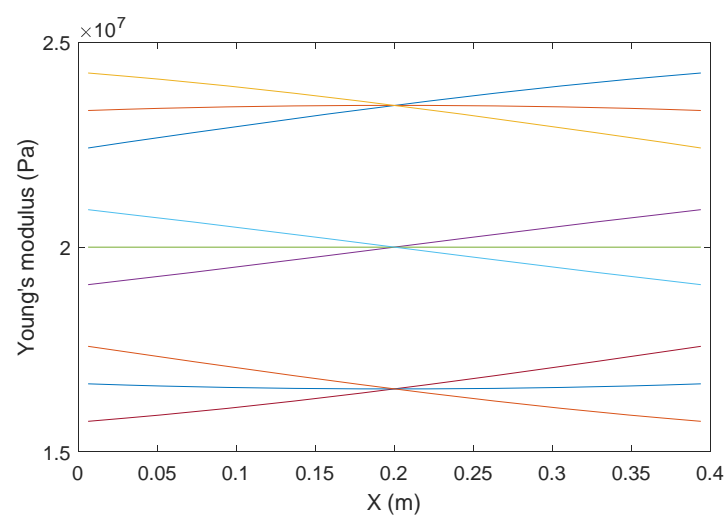

(b) The second pendulum

Figure. 15 Realization of the Young's modulus at the collocation points

The first pendulum is discretized by 3 spatial ANCF-based beam elements, while the second pendulum is discretized by 4 spatial ANCF-based beam elements. Solving the system by using the generalized- $\boldsymbol{\alpha}$ method, the configuration of the double pendulum from $0 \mathrm{~s}$ to $1.2 \mathrm{~s}$ under the deterministic assumption (i.e. $\mathrm{G}=2 \times 10^{7} \mathrm{~Pa}$ ) is shown in Fig. 16(a), while the Fig. 16(b) shows the configuration under uncertain condition.

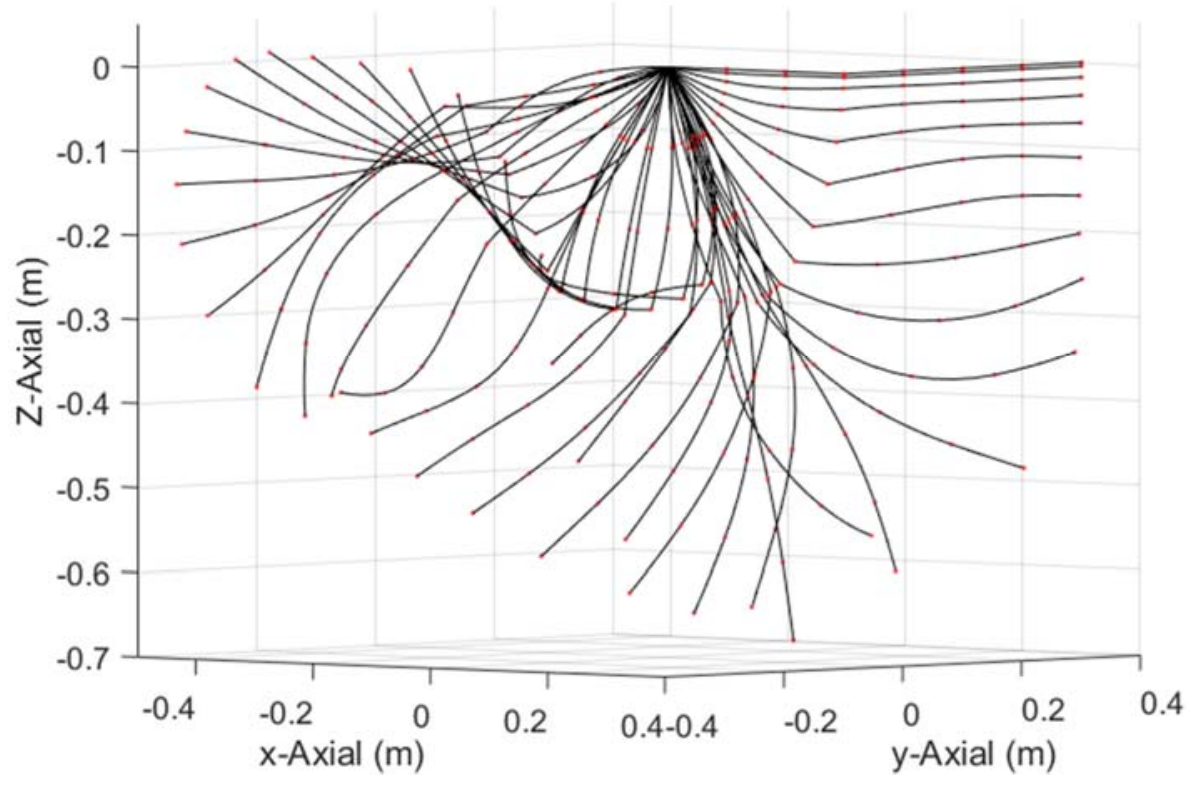

(a) Deterministic case 


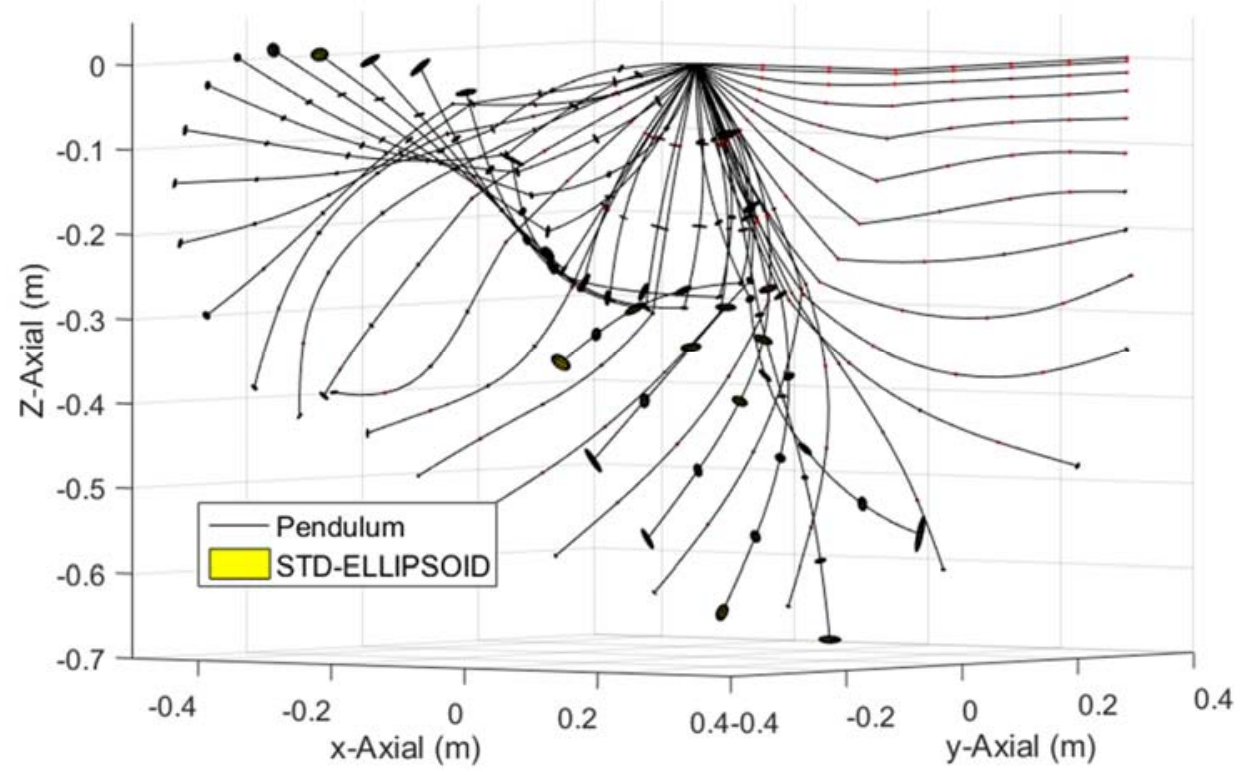

(b) Uncertain case

Figure. 16 Configuration of double space pendulum

We can find that, in the initial stage, the "ellipsoid" is too small to be seen clearly. However, with time increasing the "ellipsoid" of the nodal displacement becomes larger and larger, so uncertainty extent of system response increases with the time. To show the difference between the results of deterministic case and the uncertain case, the tracks of the end point of two pendulums are plot in Fig. 17 and 18, respectively.

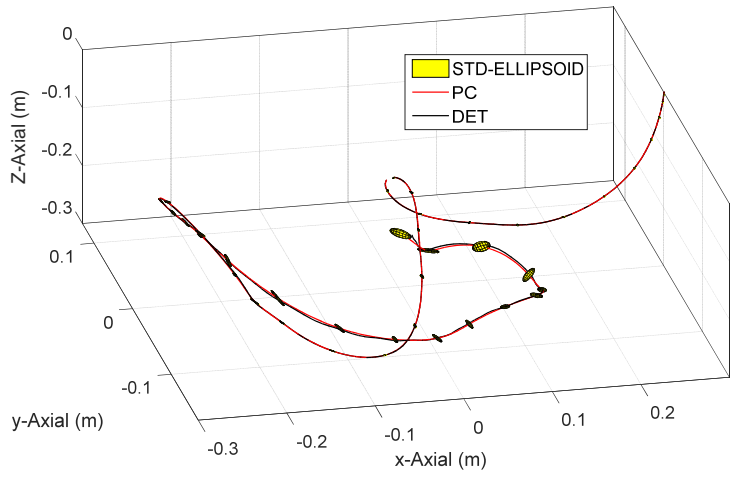

(a) Full view

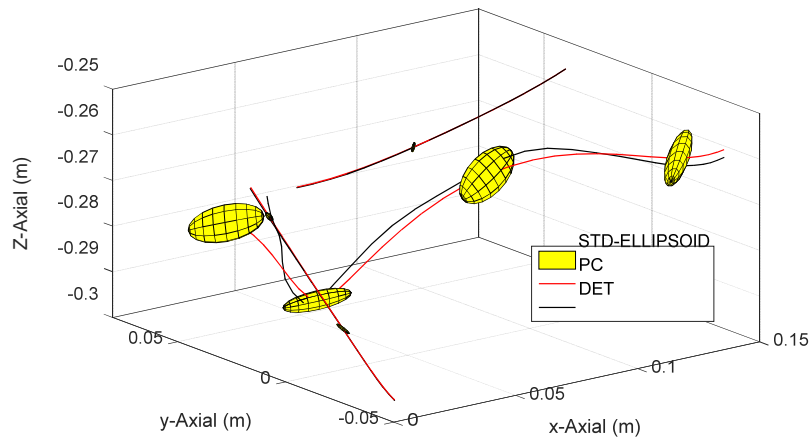

(b) Scaled view

Figure. 17 Track of the end point of the first pendulum 


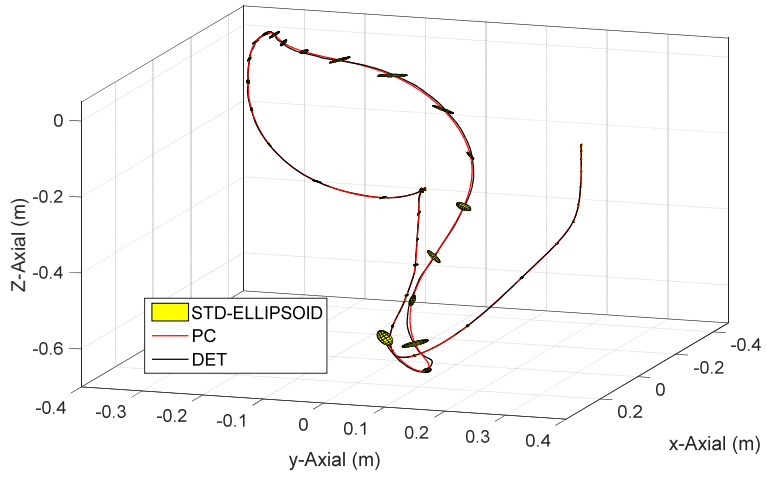

(a) Full view

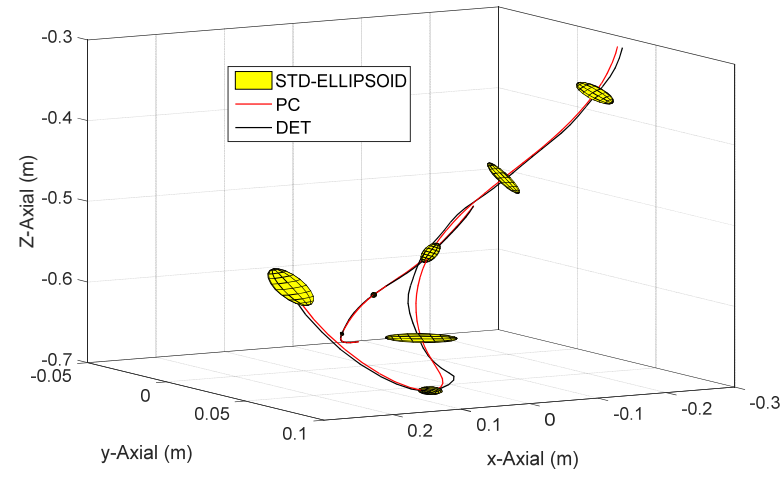

(b) Scaled view

Figure. 18 Track of the end point of the second pendulum

The solution of deterministic case is under the condition that the Young's modulus is set to the mean value of random field $2 \times 10^{7} \mathrm{~Pa}$, but it is quite different from the mean solution of that the Young's modulus is considered as random field. Therefore, it is necessary to consider the uncertainty of material properties. On the other hand, the mean of input does not guarantee to produce the mean of response. The ellipsoid in the figures can indicate the dependence of the displacement among different direction.

To validate the PC expansion, the Monte Carlo method using 1000 samples will be employed. The 1000 realizations of the Young's modulus are provided in Fig. 19. The random field of the first pendulum is discretized to only one random variable, so its realization is smoother than that of the second pendulum that is discretized to two random variables.

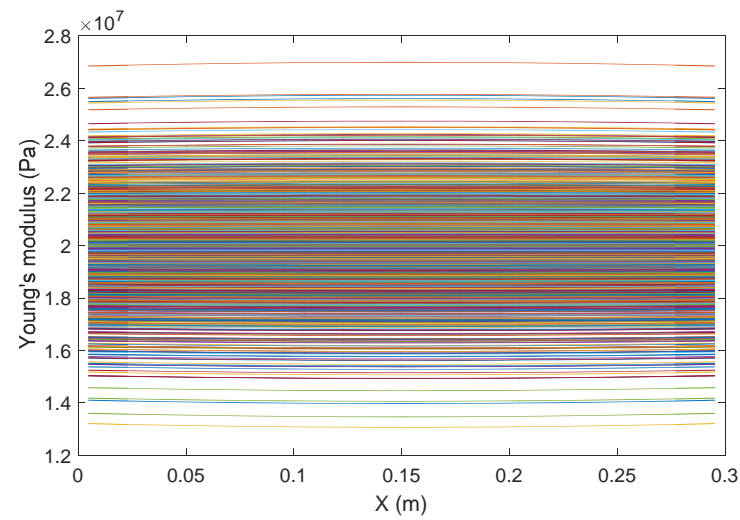

(a) The first pendulum

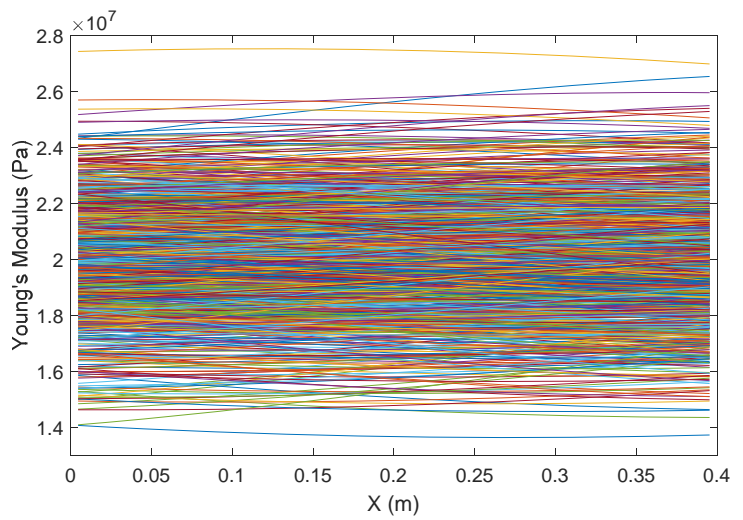

(b) The second pendulum

Figure. 19 Realizations of the Young's modulus used by Monte Carlo simulation 


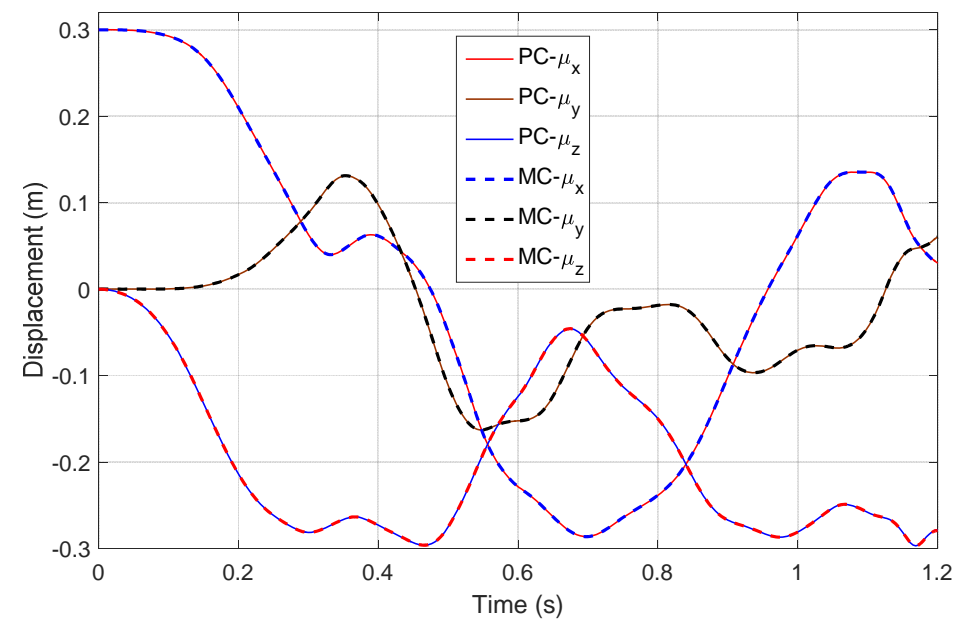

(a) The first pendulum

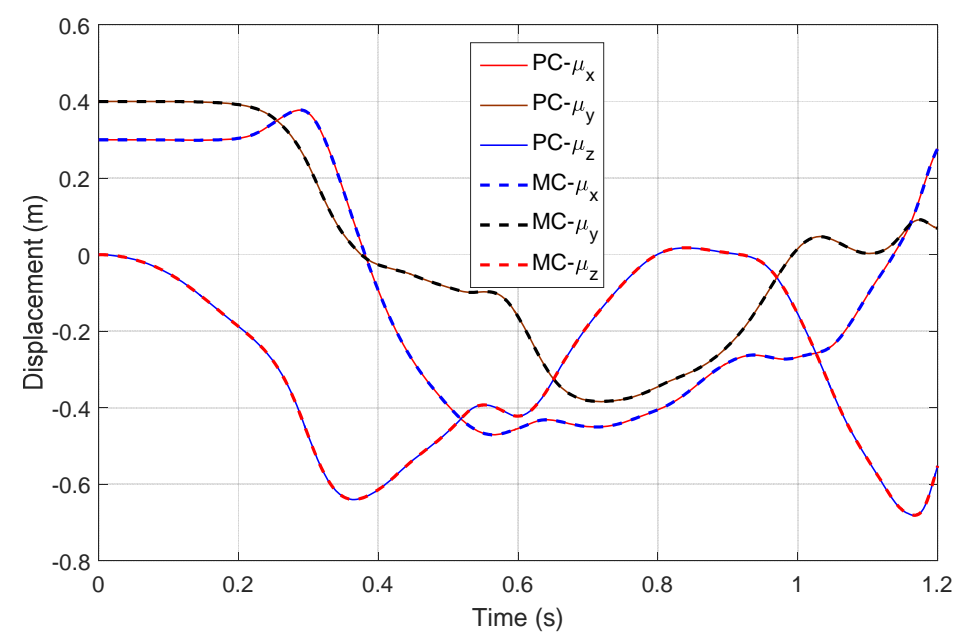

(b) The second pendulum

Figure. 20 Mean displacement of the end points

The mean displacements of the two end points are provided in Fig. 20. It can be found that the displacement of both two end points obtained by the PC expansion is coincident with that of Monte Carlo simulation. Therefore, the PC expansion has very high accuracy in estimating the mean displacement.

The displacement covariance of the first end point and the second end point are shown in Fig. 21 and 22, respectively. The covariance obtained by the PC expansion is also very close to that of Monte Carlo method. For the end point of the first pendulum, the diagonal entries $\left(\sigma_{x}^{2}, \sigma_{y}^{2}, \sigma_{z}^{2}\right)$ of the covariance matrix dominated the covariance, which means that the dependence among the three coordinates is not very strong. However, for the end point of the second pendulum, the diagonal entries of the covariance matrix are close to the magnitude of the non-diagonal entries $\left(\sigma_{x y}, \sigma_{y z}, \sigma_{x z}\right)$, which indicate that there are strong coupling among displacement of the three coordinates. 


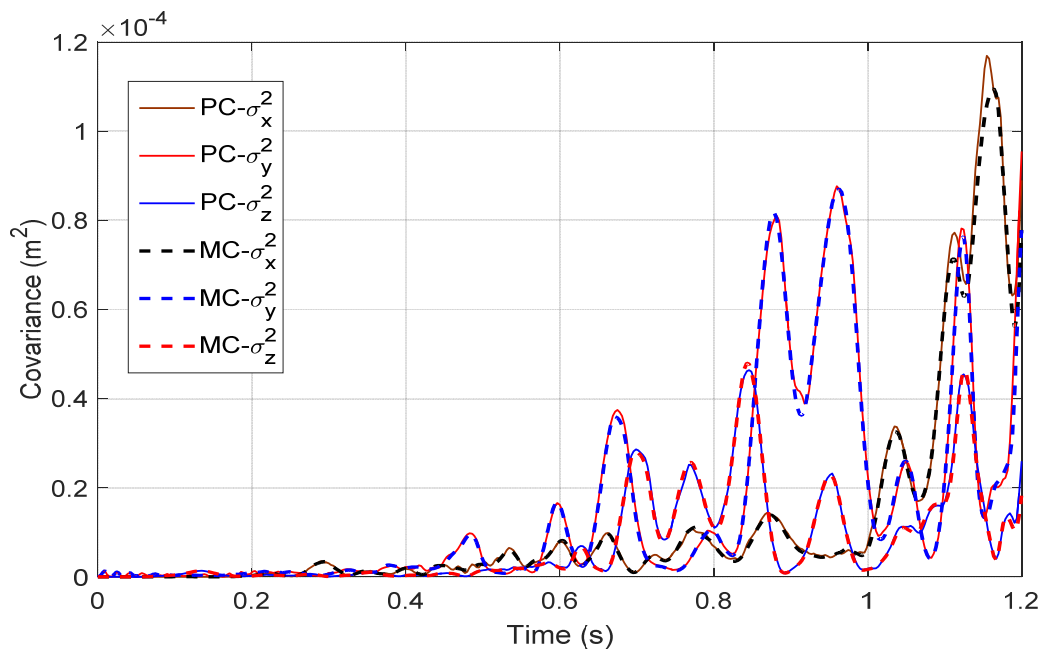

(a) The first pendulum

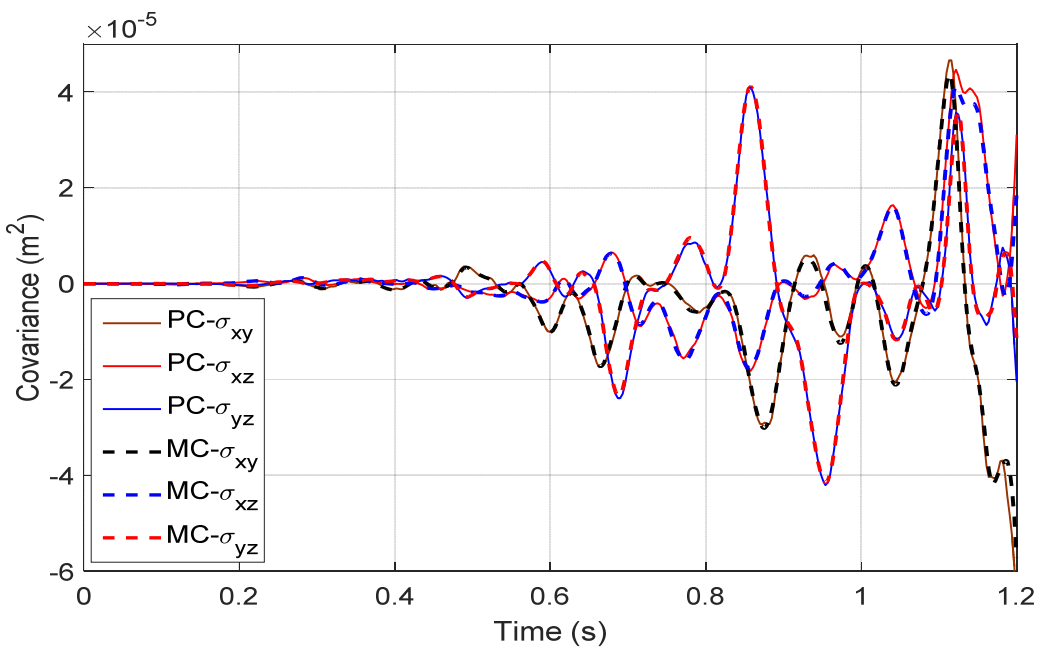

(b) The second pendulum

Figure. 21 Displacement covariance of the end point of the first pendulum

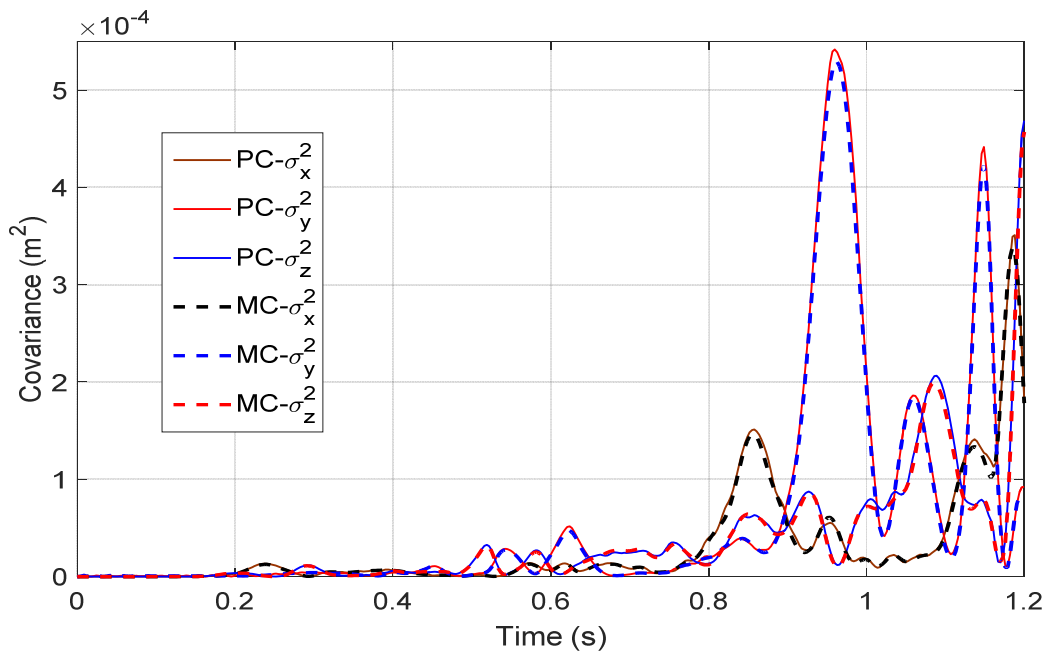

(a) The first pendulum 


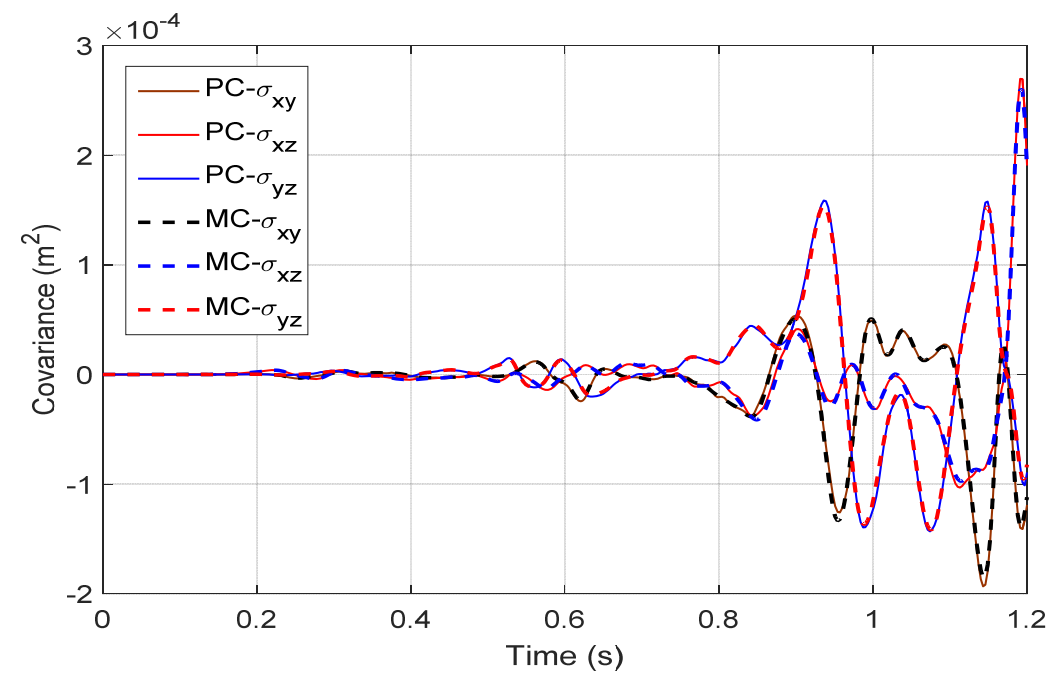

(b) The second pendulum

Figure. 22 Displacement covariance of the end point of the second pendulum

Figure 23 shows the normalized standard deviation of the displacement of the two end points. The normalized standard deviation becomes large with time increasing, so it is hard to accurately predict the position of the end point after some time, even the uncertainty extent of material properties is not large. The standard deviation of the second end point is larger than the first one, so the longer pendulum is, the larger uncertainty extent occurs. Compared the result of PC expansion to the Monte Carlo simulation, it shows that they match well, so the PC expansion is accurate. At the same time, the PC expansion only runs the flexible multibody dynamic model 27 times, which is much less than the 1000 simulations used in the Monte Carlo method, so the efficiency of PC expansion is much higher.

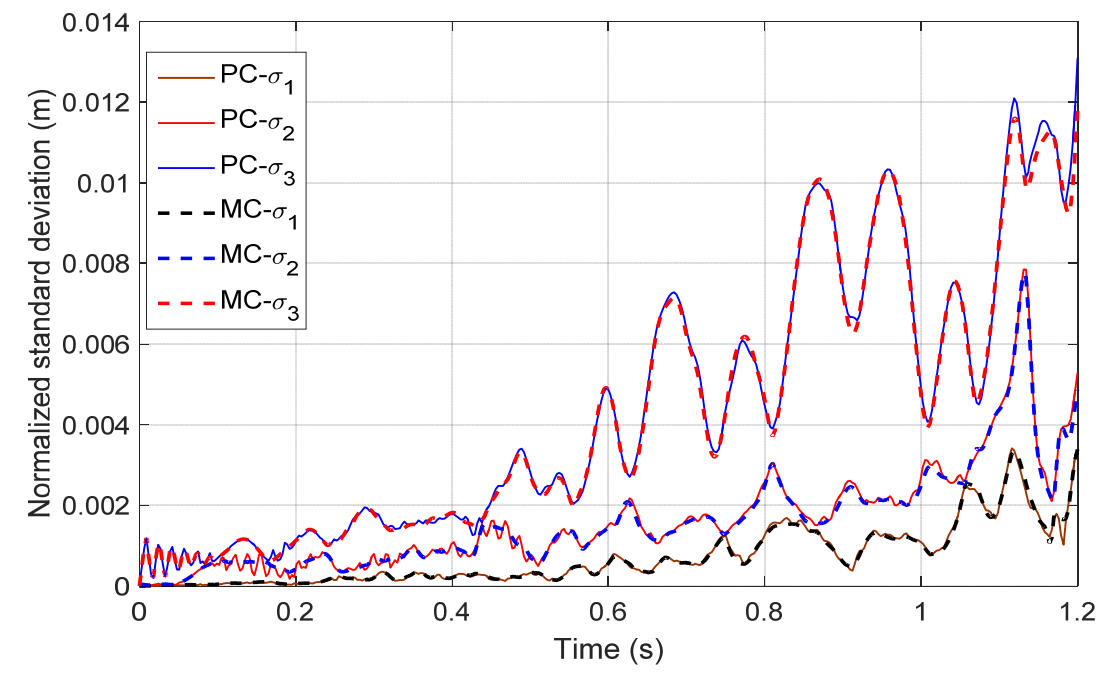

(a) The first pendulum 


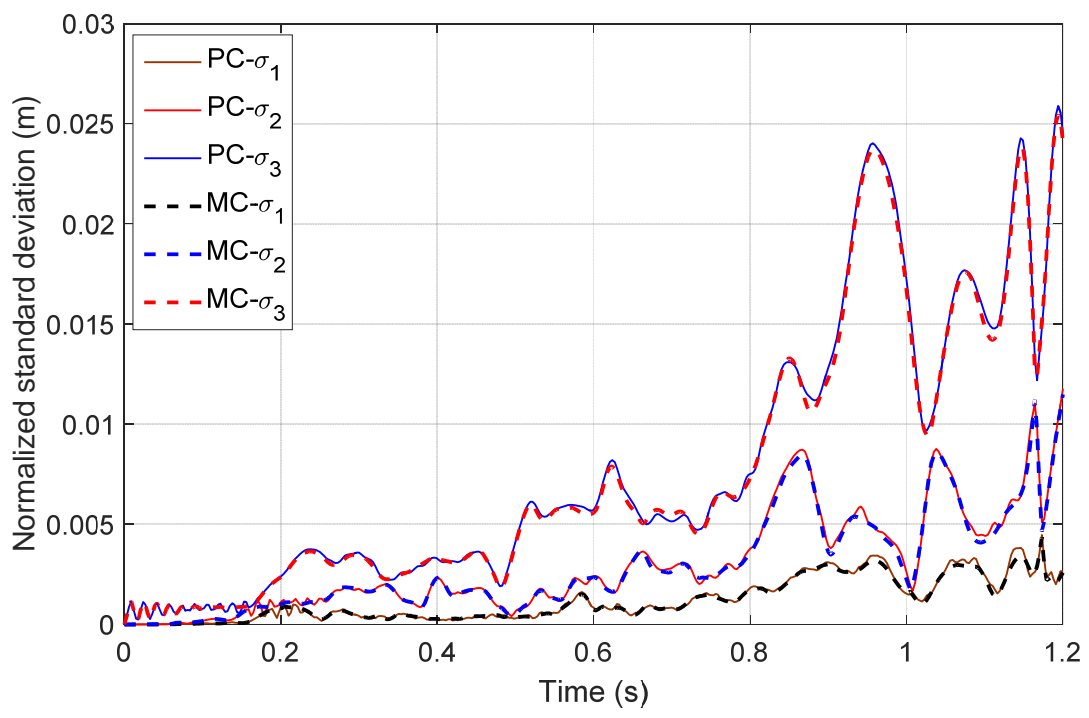

(b)The second pendulum

Figure. 23 Normalized standard deviation of end point displacement

\section{Conclusion}

This paper investigates the dynamic response of the flexible multibody system with uncertain material properties including the Young's modulus and Poisson's ratio, where the dynamic model is built by using the ANCF-based method. The uncertain material properties vary in the space domain, so they have to be described by using the random field. In numerical implementation, the Gaussian random field is discretized to several independent normal random variables by using the high accurate EOLE method. After the discretization, the PC expansion method is used to approximate the uncertain solution by a truncated Hermite series. The PC expansion is realized through the collocation method, in which the collocation points are the roots of Hermite polynomials. We prove that the collocation method produce the same coefficients as the Gaussian quadrature method. In the uncertainty computation of flexible multibody system, the traditional solver (e.g. the generalized-a method used in this paper) is not modified, but only a pre-process and a post-process are added. Since this method is a non-intrusive method, it can also be used to other elements and other numerical solver. The pre-process computes the stiffness matrix at the given collocation points, while the post-process computes the mean, covariance, and normalized standard deviation of response. The uncertain displacement is visually represented by the ellipse and ellipsoid. Two numerical examples are used to show the effectiveness of the proposed method. The results indicate that there is an obvious difference between the response of the deterministic and uncertain cases. 
The Monte Carlo method is employed to validate the PC expansion method, which shows that they have almost the same response for both the mean and covariance. However, the PC expansion method only requires 36 and 27 simulations that are much less than the 1000 simulations required in the Monte Carlo method.

\section{Acknowledgements}

This research is supported in part by the National Natural-Science-Foundation of China (11472112; 11502083), and China Postdoctoral Science Foundation (2014M562014), and Australian Research Council (Discovery Projects) (DP150102751).

\section{References}

1. Shabana, A.A.: Computational Dynamics. John Wiley \& Sons, New York (2001)

2. Shabana, A.A.: Flexible multi-body dynamics review of past and recent developments. Multibody Syst. Dyn. 1, 189-222 (1997)

3. Shabana, A.A.: An absolute nodal coordinates formulation for the large rotation and deformation analysis of flexible bodies, University of Illinois, Chicago (1997)

4. Shabana, A.A.: Definition of the slopes and absolute nodal coordinate formulation. Multibody Syst. Dyn. 1, 339-348 (1997)

5. Shabana, A.A.: Dynamics of Multibody Systems. Cambridge University Press, New York (2005)

6. Eberhard, P., Schiehlen, W.: Computational dynamics of multibody systems history, formalisms, and applications. J. Comput. Nonlinear Dyn. 1, 3-12 (2006)

7. Kerkkänen, K.S., García-Vallejo, D., Mikkola, A.M.: Modeling of Belt-Drives Using a Large Deformation Finite Element Formulation. Nonlinear Dyn. 43(3), 239-256 (2006)

8. Dufva, K., Kerkkanen, K., Maqueda, L.G., Shabana, A.A.: Nonlinear dynamics of three-dimensional belt drives using the finite-element method. Nonlinear Dyn. 48(4), 449-466 (2007)

9. Gerstmayr, J., Shabana, A.A.: Analysis of Thin Beams and Cables Using the Absolute Nodal Co-ordinate Formulation. Nonlinear Dyn. 45(1-2), 109-130 (2006)

10. Zhang, Y.Q., Tian, Q., Chen, L.P., Yang, J.Z.: Simulation of a viscoelastic flexible multibody system using absolute nodal coordinate and fractional derivative methods. Multibody Syst. Dyn. 21(3), 281-303 (2009)

11. Tian, Q., Zhang, Y.Q., Chen, L.P., Yang, J.Z.: Simulation of planar flexible multibody systems with clearance and lubricated revolute joints. Nonlinear Dyn. 60, 489-511 (2010)

12. Tian, Q., Liu, C., Machado, M., Flores, P.: A new model for dry and lubricated cylindrical joints with clearance in spatial flexible multibody systems. Nonlinear Dyn. 64(1-2), 25-47 (2011)

13. Liu, C., Tian, Q., Hu, H.Y.: Dynamics of a large scale rigid-flexible multibody system composed of composite laminated plates. Multibody Syst. Dyn. 26(3), 283-305 (2011)

14. Zhao, J., Tian, Q., Hu, H.Y.: Modal Analysis of a Rotating Thin Plate via Absolute Nodal Coordinate Formulation. J. Comput. Nonlinear Dyn. 6(4), 041013 (2011)

15. Gerstmayr, J., Matikainen, M.K., Mikkola, A.M.: A geometrically exact beam element based on the absolute nodal coordinate formulation. Multibody Syst. Dyn. 20(4), 359-384 (2008) 
16. Liu, C., Tian, Q., Hu, H.Y.: Dynamics and control of a spatial rigid-flexible multibody system with multiple cylindrical clearance joints. Mech. Mach. Theory 52, 106-129 (2012)

17. Tian, Q., Xiao, Q.F., Sun, Y.L., Hu, H.Y., Liu, H., Flores, P.: Coupling dynamics of a geared multibody system supported by ElastoHydroDynamic lubricated cylindrical joints. Multibody Syst. Dyn. 33(3), 259-284 (2014)

18. Helton, J.C., Johnson, J.D., Oberkampf, W.L., Storlie, C.B.: A sampling-based computational strategy for the representation of epistemic uncertainty in model predictions with evidence theory. Comput. Method Appl. Mech. Eng. 196, 3980-3998 (2007)

19. Dantan, J.Y., Gayton, N., Qureshi, A.J., Lemaire, M., Etienne, A.: Tolerance Analysis Approach based on the Classification of Uncertainty (Aleatory/Epistemic). Procedia CIRP 10, 287-293 (2013)

20. Kang, Z., Luo, Y.J.: Non-probabilistic reliability-based topology optimization of geometrically nonlinear structures using convex models. Comput. Method Appl. Mech. Eng. 198(41-44), 3228-3238 (2009)

21. Jiang, C., Han, X., Liu, G.P.: A sequential nonlinear interval number programming method for uncertain structures. Comput. Method Appl. Mech. Eng. 197(49-50), 4250-4265 (2008)

22. Qiu, Z.P, Ma, L.H., Wang, X.J.: Non-probabilistic interval analysis method for dynamic response analysis of nonlinear systems with uncertainty. J. Sound Vib. 319(1-2), 531-540 (2009)

23. Wu, J.L., Zhen, L., Zhang, Y.Q., Zhang, N., Chen, L.P.: Interval uncertain method for multibody mechanical systems using Chebyshev inclusion functions. Int. J. Numer. Methods Eng. 95(7), 608-630 (2013)

24. Wang, Z., Tian, Q., Hu, H.Y.: Dynamics of spatial rigid-flexible multibody systems with uncertain interval parameters. Nonlinear Dyn, DOI 10.1007/s11071-015-2504-4 (2016)

25. Ghanem, R.G., Spanos, P.D.: Stochastic Finite Elements: A Spectral Approach. Springer-Verlag, New York (1991)

26. Sudret, B., Der Kiureghian, A.: Stochastic finite element methods and reliability: a state-of-the-art report. University of California, Berkeley (2000)

27. Zhang, J., Ellingwood, B.: Orthogonal series expansion of random fields in reliability analysis. J. Eng. Mech. 120, 2660-2677 (1994)

28. Li, C.C., Der Kiureghian, A.: Optimal discretization of random field. J. Eng. Mech. 119, 1136-1154 (1993)

29. Fishman, G.S.: Monte Carlo: Concepts, Algorithms, and Applications. Springer-Verlag, New York (1996)

30. Xiu, D.B., Karniadakis, G.E.: Modeling uncertainty in steady state diffusion problems via generalized polynomial chaos. Comput. Method Appl. Mech. Eng. 191, 4927-4948 (2002)

31. Xiu, D.B., Karniadakis, G.E.: Modeling uncertainty in flow simulations via generalized polynomial chaos. J. Comput. Phys. 187(1), 137-167 (2003).

32. Li, L., Sandu, C.: On the impact of cargo weight, vehicle parameters, and terrain characteristics on the prediction of traction for off-road vehicles. J. Terramech. 44, 221-238 (2007)

33. Sandu, C., Sandu, A., and Blanchard, E.D.: Polynomial chaos-based parameter estimation methods applied to a vehicle system. Proc. Inst. Mech. Eng. K J. Multi-body Dyn. 224(1), 59-81 (2010)

34. Sarkar, A., Ghanem, R.: Mid-frequency structural dynamics with parameter uncertainty. Comput. Method Appl. Mech. Eng. 191, 5499-5513 (2002)

35. Lucor, D., Enaux, C., Jourdren, H., Sagaut, P.: Stochastic design optimization: Application to reacting flows. Comput. Method Appl. Mech. Eng. 196(49-52), 5047-5062 (2007) 
36. Batou, A., Soize, C.: Rigid multibody system dynamics with uncertain rigid bodies. Multibody Syst. Dyn. 27(3), 285-319 (2011)

37. Schmitt, K.P., Anitescu, M., Negrut, D.: Efficient sampling for spatial uncertainty quantification in multibody system dynamics applications. Int. J. Numer. Methods Eng. 80(5), 537-564 (2009)

38. Sandu, A., Sandu, C., Ahmadian, M.: Modeling Multibody Systems with Uncertainties. Part I: Theoretical and Computational Aspects. Multibody Syst Dyn. 15(4), 369-391 (2006)

39. Sandu, C., Sandu, A., Ahmadian, M.: Modeling multibody systems with uncertainties. Part II: Numerical applications. Multibody Syst. Dyn. 15(3), 241-262 (2006)

40. Wang, S.X., Wang, Y.H., He, B.Y.: Dynamic modeling of flexible multibody systems with parameter uncertainty. Chaos Solitons Fractals 36(3), 605-611 (2008)

41. Omar, A., Shabana, A.A.: A two-dimensional shear deformable beam for large rotation and deformation problems. J. Sound Vib. 243, 565-576 (2001)

42. Shabana, A.A., Yakoub, R.Y.: Three-dimensional absolute nodal coordinate formulation for beam elements: theory. J. Mech. Des. 123, 606-613 (2001)

43. García-Vallejo, D., Mayo, J., Escalona, J.L., Dominguez, J.: Efficient Evaluation of the Elastic Forces and the Jacobian in the Absolute Nodal Coordinate Formulation. Nonlinear Dyn. 35, 313-329 (2004)

44. Shabana, A.A.: Computational Continuum Mechanics. Cambride University Press, New York (2008)

45. Vanmarcke, E.: Random Fields Analysis and Synthesis. World Scientific (2010)

46. Xiu, D.B.: Numerical methods for stochastic computations: a spectral method approach.Princeton: Princeton University Press (2010)

47. Abramowitz, M., Stegun, I.: Handbook of Mathematical Functions with Formulas, Graphs, and Mathematical Tables: Dover Publications (1964)

48. Isukapalli, S.S., Roy, A., Georgopoulos, P.G.: Stochastic Response Surface Methods (SRSMs) for uncertainity propagation application to environmental and biological systems. Risk Anal. 18(3), 351-363 (1998)

49. Tian, Q., Chen, L.P., Zhang, Y.Q., Yang, J.Z.: An Efficient Hybrid Method for Multibody Dynamics Simulation Based on Absolute Nodal Coordinate Formulation. J. Comput. Nonlinear Dyn. 4(2), 021009 (2009)

50. Arnold, M., Brüls, O.: Convergence of the generalized- $\alpha$ scheme for constrained mechanical systems. Multibody Syst. Dyn. 18(2), 185-202 (2007) 